\title{
Invariances, Laplacian-Like Wavelet Bases, and the Whitening of Fractal Processes
}

\author{
Pouya Dehghani Tafti, Member, IEEE, Dimitri Van De Ville, Member, IEEE, and Michael Unser, Fellow, IEEE
}

\begin{abstract}
In this contribution, we study the notion of affine invariance (specifically, invariance to the shifting, scaling, and rotation of the coordinate system) as a starting point for the development of mathematical tools and approaches useful in the characterization and analysis of multivariate fractional Brownian motion ( $\mathrm{fBm})$ fields. In particular, using a rigorous and powerful distribution theoretic formulation, we extend previous results of Blu and Unser (2006) to the multivariate case, showing that polyharmonic splines and $\mathrm{fBm}$ processes can be seen as the (deterministic vs stochastic) solutions to an identical fractional partial differential equation that involves a fractional Laplacian operator. We then show that wavelets derived from polyharmonic splines have a behavior similar to the fractional Laplacian, which also turns out to be the whitening operator for $\mathrm{fBm}$ fields. This fact allows us to study the probabilistic properties of the wavelet transform coefficients of fBm-like processes, leading for instance to ways of estimating the Hurst exponent of a multiparameter process from its wavelet transform coefficients. We provide theoretical and experimental verification of these results. To complement the toolbox available for multiresolution processing of stochastic fractals, we also introduce an extended family of multidimensional multiresolution spaces for a large class of (separable and nonseparable) lattices of arbitrary dimensionality.
\end{abstract}

Index Terms-Affine invariance, fractional Brownian motion (fBm), fractional partial differential equations, Hurst exponent, lattices, multidimensional wavelets, operator wavelets, polyharmonic splines, whitening.

\section{INTRODUCTION}

$\mathbf{T}$ HE notion of invariance plays a significant role in mathematical modeling. The development of fractals, for instance, is entirely based on the idea of self-similarity (i.e., scaleinvariance up to a scalar factor) [1], [2]. This self-similarity can be deterministic - in which case we are led to deterministic fractals such as the famous Koch snowflake, or the elaborate Mandelbrot set-but it can also be understood in a statistical sense-leading to stochastic fractals, the prime examples of which are fractional Brownian motion ( $\mathrm{fBm}$ ) processes [3] (see also Chainais et al. [4] for a generalization based on the notion of scaling).

Fractional Brownian motion models generalize Lévy's Brownian motion [5] of Gaussian type. These processes have

Manuscript received October 09, 2007; revised October 20, 2008. Current version published March 13, 2009. This work was supported in part by the Swiss Science Foundation. This work was supported by the Swiss Science Foundation. The associate editor coordinating the review of this manuscript and approving it for publication was Prof. Minh N. Do.

The authors are with the Biomedical Imaging Group,École Polytechnique Fédérale de Lausanne (EPFL), Station 17, CH-1015 Lausanne, Switzerland (e-mail: p.d.tafti@ieee.org; dimitri.vandeville@epfl.ch; michael.unser@epfl.ch).

Digital Object Identifier 10.1109/TIP.2008.201145 long been associated with the phenomenon of long-range dependence and $1 / f^{\alpha}$-like power spectra that frequently appear in areas as diverse as hydrology, financial mathematics, network traffic analysis, terrain modeling, and image processing [1], [6]-[8]. In the case of the latter, the relevance of fBm processes in modeling images has been claimed on the basis of observations of scale-invariance and the associated power-law spectra in natural images [9]-[11].

A multivariate $\mathrm{fBm}$ field $\mathfrak{B}_{H}$ is a nonstationary Gaussian process $^{1}$ identified by a single parameter $0<H<1$ - the Hurst parameter, after Harold Edwin Hurst (1880-1978), for his seminal contribution to the study of such processes in the context of hydrology [2], [12] — that characterizes its covariance up to a scalar normalization factor

$$
\mathbf{E}\left\{\mathfrak{B}_{H}(\boldsymbol{x}) \mathfrak{B}_{H}\left(\boldsymbol{x}^{\prime}\right)\right\} \propto\|\boldsymbol{x}\|^{2 H}+\left\|\boldsymbol{x}^{\prime}\right\|^{2 H}-\left\|\boldsymbol{x}-\boldsymbol{x}^{\prime}\right\|^{2 H} .
$$

Estimation of the Hurst parameter is important in practical applications, and is, e.g., used in image processing to classify different types of texture based on their second order statistics [13], [14].

Multiresolution analysis [15], [16] was identified early on in its development as a decidedly effective tool for the study of self-similarity [17]-[26]. Its utility in the estimation of parameters of self-similar processes (especially in the 1-D setting and for estimating the Hurst parameter) is, therefore, well documented [20], [27]-[29]. The essential observation in this regard is that the logarithm of the wavelet energy of an fBm process varies linearly with scale, with a slope that depends on the Hurst parameter $H$.

Intuitively, the above observation appears deceptively simple. After all, this would seem to be a straightforward consequence of the $1 / f^{\alpha}$-like power spectrum of $\mathrm{fBm}$ and the logarithmic spectral partitioning afforded by the wavelet transform. A rigorous derivation of this result is, however, subtler, as $\mathrm{fBm}$ - being nonstationary-does not, in fact, have a power spectrum in the classical sense.

On account of this, one of our main motivations in writing this paper has been to propose a rigorous interpretation of the spectral characterization of multivariate isotropic $\mathrm{fBm}$, in the sense of a whitening/innovation model (Section $\mathrm{V}$ ). This distributional framework, which is deduced from basic invariance principles (Section III), provides a powerful formalism for defining and analysing $\mathrm{fBm}$ and similar processes. Our results here generalize those of Blu and Unser [30] who studied the single-variable case. Operator models for self-similar fields were also studied

\footnotetext{
${ }^{1}$ In this paper, we do not distinguish between random processes and random fields, using both terms interchangeably to refer to multivariate random functions.
} 
by Benassi et al. [31], [32], who focused on the link between operators and multivariate random fields and their relation to wavelets. The 1-D analysis of Wyss [33] is also relevant.

The said formulation also links the study of fBm processes to spline theory via providing a convenient and unifying interpretation of $\mathrm{fBm}$ processes and polyharmonic splines as stochastic vs deterministic solutions to the same (fractional) partial differential equation [34]. This, in the light of the fundamental relation between splines and wavelets (Section IV), allows us to derive interesting and general results concerning the wavelet analysis of fractional Brownian motion (Section V). We for instance show the quasi-whitening effect of a polyharmonic wavelet transform on $\mathrm{fBm}$ processes.

To complement the mathematical toolset for the analysis of multivariate $\mathrm{fBm}$, we have included a comprehensive account of a general construction scheme for multidimensional polyharmonic spline multiresolution spaces, proving all essential properties for forming a multiresolution analysis. The generality of our construction (which extends the works of Rabut and Bacchelli et al. [35], [36] and Van De Ville et al. [37]) makes it suitable for multiresolution approximation in any number of dimensions and on virtually all sampling lattices of interest that display some form of isotropy.

The organization of the remainder of the paper, in brief, is as follows. In Section II, we review some mathematical preliminaries. We formalize the idea of isotropic affine invariance in Section III and use it to identify a family of fractional partial differential operators that appear in the characterization of both polyharmonic splines and $\mathrm{fBm}$ processes. The theory of multidimensional polyharmonic spline multiresolution is developed in Section IV. Next, in Section V, we provide a characterization of $\mathrm{fBm}$ based on an innovation model. We then exploit the link between splines and fBm processes in Section VI, to derive some characteristic results concerning polyharmonic wavelet analysis of $\mathrm{fBm}$. Based on these results, estimation of the Hurst parameter is also discussed and a few experimental results are provided in Section VI-B. Some final remarks conclude the paper.

\section{Mathematical Preliminaries AND Notation}

The theory of generalized random processes utilized in this paper is exposited in the works of Gel'fand et al. [38], [39]. For reference, some of the main definitions are summarized in this section. This section shall also serve to fix our basic notation and to recall some facts and definitions from the theory of lattices.

\section{A. Some Notational Conventions}

We use the MATLAB notation for row and column vectors and also follow the multi-index convention, according to which, given a vector $\boldsymbol{x}=\left[x_{1} ; \ldots ; x_{d}\right] \in \mathbb{R}^{d}$ and a multi-index $\boldsymbol{k}=$ $\left[k_{1} ; \ldots ; k_{d}\right] \in \mathbb{Z}_{\geq 0}^{d}$ (d always denotes the dimensionality of the domain)

$$
\boldsymbol{x} \stackrel{\boldsymbol{k}}{\stackrel{\text { def }}{=}} \prod_{1 \leq i \leq d} x_{i}^{k_{i}}, \quad \boldsymbol{k} ! \stackrel{\text { def }}{=} \prod_{1 \leq i \leq d} k_{i} !, \quad \text { and } \quad|\boldsymbol{k}| \stackrel{\text { def }}{=} \sum_{1 \leq i \leq d} k_{i} .
$$

Other notation is defined where first used.

\section{B. Generalized Functions}

A regular function $u$ of a variable $x \in \mathbb{R}^{d}$ is characterized by the value it assigns to its argument $\boldsymbol{x}$ (i.e., $u(\boldsymbol{x})$ for $\boldsymbol{x} \in \mathbb{R}^{d}$ ). In contrast, a generalized function or distribution $f$ is specified in terms of inner-products ${ }^{2}\langle f, u\rangle$ with test functions $u$ belonging to some inner-product space $\mathcal{K}$. Intuitively, these inner-products can be interpreted as linear observations or measurements of $f$. The advantage is that in this framework we can conceive of entities that need no longer be defined point-wise. The space of all generalized functions defined by their (bounded) inner-products with elements of $\mathcal{K}$ is identified with $\mathcal{K}^{\prime}$, the continuous dual of $\mathcal{K}$.

Given an operator A with adjoint $\mathrm{A}^{*}$, both defined on our space of test functions, we may extend the domain of $\mathrm{A}$ to the corresponding space of generalized functions $\left(\mathcal{K}^{\prime}\right)$ using the following defining identity

$$
\langle\mathrm{A} f, u\rangle \stackrel{\text { def }}{=}\left\langle f, \mathrm{~A}^{*} u\right\rangle .
$$

Thus, e.g., for the shift operator we shall have

$$
\langle f(\cdot-\boldsymbol{h}), u(\cdot)\rangle \stackrel{\text { def }}{=}\langle f(\cdot), u(\cdot+\boldsymbol{h})\rangle, \text { for all } u \in \mathcal{K} .
$$

The Fourier transform defines a one-to-one mapping between a suitably chosen space $\mathcal{K}$ of test functions and the space $\hat{\mathcal{K}}$ of their Fourier transforms. With Parseval's identity in mind, the Fourier transform of a generalized function $f(x) \in \mathcal{K}^{\prime}$ can be defined as the generalized function $\hat{f}(\boldsymbol{\omega}) \in \hat{\mathcal{K}}^{\prime}$ that satisfies the identity

$$
\langle\hat{f}(\boldsymbol{\omega}), \hat{u}(\boldsymbol{\omega})\rangle=(2 \pi)^{d}\langle f(\boldsymbol{x}), u(\boldsymbol{x})\rangle, \text { for all } u \in \mathcal{K} .
$$

If we choose $\mathcal{K}$ to be the Schwartz space of $d$-variate rapidly decaying smooth functions (denoted here by $\mathcal{S}\left(\mathbb{R}^{d}\right)$ or simply by $\mathcal{S}$ ), $\mathcal{K}$ and $\hat{\mathcal{K}}$ (and, therefore, $\mathcal{K}^{\prime}$ and $\hat{\mathcal{K}}^{\prime}$ ) coincide. A familiar example of a generalized function defined over $\mathcal{S}$ is Dirac's delta

$$
\langle\delta, u\rangle \stackrel{\text { def }}{=} u(\mathbf{0}) .
$$

The Fourier transform of $\delta(\boldsymbol{x})$ is the constant 1 , since $\langle 1, \hat{u}\rangle=$ $\int \mathrm{d} \boldsymbol{\omega} 1 \hat{u}(\boldsymbol{\omega})=(2 \pi)^{d} u(\mathbf{0})=(2 \pi)^{d}\langle\delta, u\rangle$.

\section{Generalized Random Processes and Random Fields}

To generalize the notion of a random process a similar approach may be used, where one replaces point values by inner products. Accordingly, in the stochastic analysis of Gel'fand and Vilenkin [39], a generalized random process $\mathfrak{X}$ is defined as a random generalized function, which is to say that it corresponds to a family of random variables

$$
\mathfrak{X}_{u} \stackrel{\text { def }}{=}\langle\mathfrak{X}, u\rangle, \quad u \in \mathcal{K}
$$

characterized by the consistent specification of a joint probability measure for all finite sets of test functions $u$. This should

${ }^{2}$ What we shall here refer to as an inner-product is in more accurate (but perhaps less familiar) terms a duality pairing. 
be compared with the definition of classical random processes, where point-wise random variables $\mathfrak{X}(\boldsymbol{x})$ replace the $\mathfrak{X}_{u}$ s.

Characteristic Functional: A (real-valued) generalized random process $\mathfrak{X}$ can also be described by its characteristic functional

$$
\mathbf{Z}_{\mathfrak{X}}(u) \stackrel{\text { def }}{=} \mathbf{E}\left\{\mathrm{e}^{\mathrm{j}\langle\mathfrak{X}, u\rangle}\right\}
$$

(where $\mathbf{E}$ denotes the expectation functional). The characteristic functional is continuous and positive-definite, and is equal to 1 for $u \equiv 0$. It provides a complete description of the random process $\mathfrak{X}$. This is due to the fact that

$$
\mathbf{Z}_{\mathfrak{X}}\left(\sum_{1 \leq k \leq N} \omega_{k} u_{k}\right)
$$

is a continuous and positive-definite function of $\omega_{k} \mathrm{~s}$ and, hence, by Bochner's theorem, corresponds to the Fourier transform of a probability measure- specifically, the joint probability measure of $\mathfrak{X}_{u_{1}}, \ldots, \mathfrak{X}_{u_{N}}[39$, ch. III, §2.6].

(In comparison, in the classical theory $\mathbf{E}\left\{\mathrm{e}^{\mathrm{j} \sum_{k} \omega_{k} \mathfrak{X}\left(\boldsymbol{x}_{k}\right)}\right\}$ provides the Fourier transform of the joint probability measure of $\mathfrak{X}\left(\boldsymbol{x}_{1}\right), \ldots, \mathfrak{X}\left(\boldsymbol{x}_{N}\right)$. Informally, this would correspond to choosing $\sum_{1 \leq k \leq N} \omega_{k} \delta\left(\cdot-\boldsymbol{x}_{k}\right)$ as the "test" function).

Correlation Form: The correlation form $\left\langle\langle u, v\rangle_{\mathfrak{X}}\right.$ of the (real) random process $\mathfrak{X}$ is defined as

$$
\langle\langle u, v\rangle\rangle_{\mathfrak{X}} \stackrel{\text { def }}{=} \mathbf{E}\left\{\mathfrak{X}_{u} \mathfrak{X}_{v}\right\} .
$$

The following relationship exists between the generalized correlation form $\langle\langle u, v\rangle\rangle_{\mathfrak{X}}$ and the (generalized) correlation function $c_{\mathfrak{X}}\left(\boldsymbol{x}, \boldsymbol{x}^{\prime}\right)$ of a generalized random process

$$
\langle\langle u, v\rangle\rangle_{\mathfrak{X}}=\int \mathrm{d} \boldsymbol{x} \mathrm{d} \boldsymbol{x}^{\prime} c_{\mathfrak{X}}\left(\boldsymbol{x}, \boldsymbol{x}^{\prime}\right) u(\boldsymbol{x}) v\left(\boldsymbol{x}^{\prime}\right) .
$$

In addition, for a Gaussian random process, the characteristic functional and the correlation form are related by the equation

$$
\mathbf{Z}_{\mathfrak{X}}(u)=\mathrm{e}^{-(1 / 2)\langle\langle u, u\rangle\rangle_{\mathfrak{X}}}
$$

This shows that, as expected, a Gaussian process is fully characterized by its correlation form.

\section{Lattices}

A lattice $£_{0}$ in $\mathbb{R}^{d}$ is the set of all integer linear combinations of $d$ linearly independent vectors $\boldsymbol{q}_{1}, \ldots, \boldsymbol{q}_{d}$; that is

$$
£_{0}=\mathrm{Q} \mathbb{Z}^{d}
$$

with $\mathrm{Q}=\left[\boldsymbol{q}_{1}, \ldots, \boldsymbol{q}_{d}\right][40]$, [41]. In general, there exist several generator matrices $Q$ that lead to the same lattice. Yet, they all have the same absolute determinant $|\mathrm{Q}|$ (known as the sampling density). For simplicity, we shall assume the normalization $|\mathrm{Q}|=1$.

A multidimensional lattice may be partitioned into so-called cosets that are translates of one another. This is a generalization of the concept of dividing the set of integers $\mathbb{Z}$ into even and odd numbers, or, more generally, into $k$ equivalence classes modulo $k$. In the case of lattices in $\mathbb{R}^{d}$, such a partitioning is achieved by means of a subsampling matrix $\mathrm{D}$, which plays the role of the integer $k$ in the 1-D case. $\mathrm{D}$ is an integer $d \times d$ matrix with all eigenvalues strictly greater than 1 in the absolute. It is used to define a subsampling relation for lattices

$$
£_{n+1}=\mathrm{QDQ}^{-1} £_{n} \stackrel{\text { def }}{=} \mathrm{D}_{\mathrm{Q}} £_{n} .
$$

From there

$$
£_{n}=\mathrm{QD}^{n} \mathbb{Z}^{d}=\mathrm{D}_{\mathrm{Q}}^{n} £_{0} .
$$

Similar to the partitioning of the integers modulo $k$, we find a two-scale relationship for the decomposition of $£_{n}$ into $|\mathrm{D}|$ cosets, which are translated versions of the lower resolution lattice $£_{n+1}$

$$
£_{n}=\left(\bigcup_{1 \leq i<|\mathrm{D}|} £_{n+1}+\mathrm{QD}^{n} \boldsymbol{\zeta}_{i}\right) \cup £_{n+1} .
$$

Here, the multiinteger vectors $\boldsymbol{\zeta}_{i}$-taken to be of minimum length and dubbed principal coset representatives-are specified uniquely modulo $\mathrm{D} \mathbb{Z}^{d}$.

For a given lattice hierarchy $£_{n}, n \in \mathbb{Z}$, the dual (or reciprocal) lattice hierarchy $£_{-n}^{*}$ is defined by the relation

$$
\boldsymbol{p}^{\top} \boldsymbol{q} \in \mathbb{Z}, \quad \text { for all } \boldsymbol{q} \in £_{n}, \quad \boldsymbol{p} \in £_{-n}^{*} .
$$

It follows that this hierarchy can be constructed using the matrix pair of $Q^{-\top} \stackrel{\text { def }}{=}\left(Q^{\top}\right)^{-1}$ and $D^{\top}$. Accordingly, we also define $D_{Q}^{*} \stackrel{\text { def }}{=} Q^{-\top} D^{\top} Q^{\top}$.

We define the lattice convolution operator or lattice filter corresponding to a sequence $v[\boldsymbol{k}], \boldsymbol{k} \in \mathbb{Z}^{d}$, as the operator

$$
\mathrm{V}_{\mathrm{Q}}: f(\cdot) \mapsto \sum_{\boldsymbol{k} \in \mathbb{Z}^{d}} v[\boldsymbol{k}] f(\cdot-\mathrm{Q} \boldsymbol{k}) .
$$

Its Fourier expression is

$$
\hat{\mathrm{V}}_{\mathrm{Q}}(\boldsymbol{\omega})=\sum_{\boldsymbol{k} \in \mathbb{Z}^{d}} v[\boldsymbol{k}] \mathrm{e}^{-\mathrm{j} \boldsymbol{k}^{\top} Q^{\top} \boldsymbol{\omega}} .
$$

Conversely, those and only those operators with Fourier expressions that can be written in the above form represent lattice convolutions. These Fourier expressions are in effect those that are $2 \pi £_{0}^{*}$-periodic (i.e., $2 \pi \boldsymbol{p}$-periodic for any $\boldsymbol{p} \in £_{0}^{*}$ ).

We also have a lattice version of the Poisson formula

$$
\mathcal{F}\left\{\sum_{\boldsymbol{k} \in \mathbb{Z}^{d}} \delta(\boldsymbol{x}-\mathrm{Q} \boldsymbol{k})\right\}=\frac{(2 \pi)^{d}}{|\mathrm{Q}|} \sum_{\boldsymbol{k} \in \mathbb{Z}^{d}} \delta\left(\boldsymbol{\omega}-2 \pi \mathrm{Q}^{-\top} \boldsymbol{k}\right) .
$$

Remark 1: The families of multiscale lattices that we shall consider in this work are restricted in two ways.

LAT-1. First, for our multiresolution construction we are interested in self-similar multiscale lattices. This means that the lattice coarsening matrix $D_{Q}$-and, consequently, its dual $\mathrm{D}_{\mathrm{Q}}^{*}$ - should correspond to similarity transforms. LAT -2 . Second, we require the existence of a $d \times N$ integer matrix $Y \stackrel{\text { def }}{=}\left[\boldsymbol{y}_{1}, \ldots, \boldsymbol{y}_{N}\right](N \geq d)$, such that the lattice 
vectors $\mathrm{Q} \boldsymbol{y}_{1}, \ldots, \mathrm{Q} \boldsymbol{y}_{N}$ generate $£_{0}$, and constitute a tight frame for $\mathbb{R}^{d}$. The latter is equivalent to requiring that

$$
\mathrm{QYY}^{\top} \mathrm{Q}^{\top}=\sum_{1 \leq i \leq N} \mathrm{Q} y_{i} \boldsymbol{y}_{i}^{\top} \mathrm{Q}^{\top}=\mu^{2} \mathbf{I}
$$

for some scalar $\mu$. We furthermore assume $\left\{\mathrm{Q} \boldsymbol{y}_{i}\right\}$ to be simple, i.e., not to contain any pair of linearly dependent vectors.

We note that for any lattice, there exist infinitely many subsampling schemes that satisfy the first requirement. In addition, the second requirement is satisfied by virtually all lattices that are typically used in multidimensional multiresolution signal processing (such as the Cartesian, quincunx, and hexagonal lattices in $\mathbb{R}^{2}$, and the Cartesian, FCC, and BCC lattices in $\mathbb{R}^{3}$ ). For instance, for the Cartesian and quincunx lattices in $\mathbb{R}^{2}$ (both with $\mathrm{Q}=[1,0 ; 0,1])$, the matrices

$$
Y=\left[\begin{array}{ll}
1 & 0 \\
0 & 1
\end{array}\right] \quad \text { and } \quad Y^{\prime}=\left[\begin{array}{cccc}
1 & 0 & 1 & -1 \\
0 & 1 & 1 & 1
\end{array}\right]
$$

provide two examples of such systems. A similar system for the hexagonal lattice (with $Q \propto[1,0.5 ; 0, \sqrt{3} / 2]$ ) uses the matrix

$$
Y^{\prime \prime}=\left[\begin{array}{ccc}
1 & 0 & 1 \\
0 & 1 & -1
\end{array}\right]
$$

\section{OPERATORS AND INVARIANCES}

The fundamental observation that underlies this work is that we can characterize specific classes of splines and stochastic processes as solutions to a fractional partial differential equation of the form

$$
\mathrm{U}\{\text { solution }\}=\text { driving term }
$$

where $\mathrm{U}$ is a fractional partial differential operator with certain properties, and the driving term is either a sum of Dirac deltas (in the deterministic formulation, leading to U-splines) or a white Gaussian noise process (in the stochastic formulation, leading to random processes whitened by $\mathrm{U}$ ).

In this section we shall use invariance principles to define a particular family of such fractional partial differential operators that produce polyharmonic splines (Section IV) as deterministic solutions and also characterize isotropic multidimensional fractional Brownian motion (Section V) in the stochastic setting.

The link between the deterministic and stochastic formulations is later explored in Section VI, where we investigate the properties of polyharmonic wavelet analysis of fractional Brownian motion.

\section{A. Scale- and Rotation-Invariant Operators}

The invariances we shall consider are those under the scaling, shifts, and rotations of the coordinate system [1], with the first leading to self-similar fractal structures, and the latter two relieving us from the - uncomfortable and often arbitrary — choice of an origin and a set of preferred directions.

Specifically, we shall study a family of convolution operators with continuous Fourier expressions, which, in addition to shift-invariance (intrinsic to convolution), have the following invariance properties.

INV-1. Scale-invariance: The operators of interest commute with scaling operators (up to a constant that may vary continuously with scale) in order to allow multiscale constructions. In mathematical notation, we want

$$
\mathrm{U} \circ \mathrm{S}_{a}=\alpha(a) \mathrm{S}_{a} \circ \mathrm{U}
$$

where $\mathrm{S}_{a}: f(\boldsymbol{x}) \mapsto f\left(a^{-1} \boldsymbol{x}\right), a>0$, represents the scaling operator and $\alpha(a)$ is a strictly positive continuous function.

INV-2. Rotation-invariance: The operators are in addition invariant under rotations of the coordinate system and, therefore, lead to isotropic models. In other words, the operators commute with rotations about the origin

$$
\mathrm{U} \circ \mathrm{R}_{\boldsymbol{\theta}}=\mathrm{R}_{\boldsymbol{\theta}} \circ \mathrm{U} .
$$

The following is a known result in the context of rotationand scale-invariant quadratic functionals (in this case, $Q(f) \stackrel{\text { def }}{=}$ $\left.\|\mathrm{Uf}\|_{2}^{2}\right)$ [42]-[44].

Theorem 1: The (per assumption continuous) Fourier expression of a real operator $\mathrm{U}$ fulfilling requirements INV-1 and INV-2 has the following form for some $\gamma \geq 0$

$$
\hat{\mathrm{U}}(\boldsymbol{\omega})=c\|\boldsymbol{\omega}\|^{2 \gamma} \text {. }
$$

The normalized version of such an operator (with $c=1$ ), which we denote by $\Delta^{\gamma}$, can be considered the $\gamma$ th real (fractional) iterate of the Laplacian (albeit discarding a factor of $\left.(-1)^{\gamma}\right)$. The following are easy to check

$$
\Delta^{0}=\text { identity; } \quad \Delta^{\gamma} \Delta^{\gamma^{\prime}}=\Delta^{\gamma+\gamma^{\prime}} .
$$

The fractional Laplacian has a nontrivial null-space and, as a result, infinitely many inverses differing in terms from the nullspace.

Remark 2: The null-space includes, for instance, certain functions with (generalized) Fourier transforms concentrated at the origin (i.e., at $\boldsymbol{\omega}=\mathbf{0}$ ). Since any such generalized Fourier symbol can be written as a finite sum of derivatives of $\delta(\boldsymbol{\omega})[45$, ch. II, $\$ 4.5$, p. 119, Theorem], the corresponding members of the null-space are polynomial functions up to a certain degree. This, however, is not a complete characterization of the null-space in general.

\section{B. Inverse Operators}

Looking back at (7), one may be tempted to define the inverse of $\Delta^{\gamma}$ as the operator $\Delta^{-\gamma}$ with the Fourier expression

$$
\|\omega\|^{-2 \gamma} \text {. }
$$

It is immediately noticed, however, that this Fourier form has a nonsummable singularity at the origin for $2 \gamma \geq d$; therefore, in general, the integral

$$
\Delta^{-\gamma} f(\boldsymbol{x})=(2 \pi)^{-d} \int_{\mathbb{R}^{d}} \mathrm{~d} \boldsymbol{\omega} \mathrm{e}^{\mathrm{j} \boldsymbol{x}^{\top} \boldsymbol{\omega}}\|\boldsymbol{\omega}\|^{-2 \gamma} \hat{f}(\boldsymbol{\omega})
$$


needs to be properly interpreted, i.e., regularized. ${ }^{3}$ Since regularization can be done in more than one way, $\Delta^{-\gamma}$ in fact represents a family of inverses rather than a single one.

Different regularizations essentially correspond to different (boundary or other) linear constraints on the solution of a fractional differential equation of the form

$$
\Delta^{\gamma} \rho(\boldsymbol{x})=f(\boldsymbol{x}) .
$$

These constraints may be satisfied by adding an appropriate term from the null-space of $\Delta^{\gamma}$ to a particular solution.

One of the possible inverse operators is the left inverse (introduced by Blu and Unser in the single-variable setting [30]; denoted by $\Delta^{-\gamma}$ here), which is obtained by removing a sufficient number of lower order terms from the Taylor series expansion of $\hat{f}(\boldsymbol{\omega})$ at the origin

$$
\begin{aligned}
& \grave{\Delta}^{-\gamma} f(\boldsymbol{x}) \stackrel{\text { def }}{=}(2 \pi)^{-d}
\end{aligned}
$$

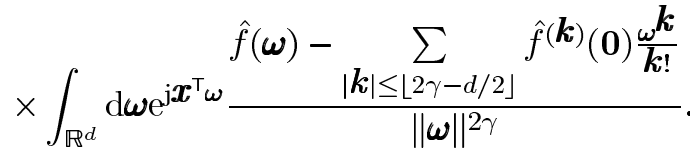

It can be checked that

$$
\grave{\Delta}^{-\gamma} \Delta^{\gamma} f=f
$$

for any $f \in \mathcal{S}$, hence the name left inverse.

The adjoint of $\grave{\Delta}^{-\gamma}$ over $\mathcal{S}$ is the operator $\Delta^{-\gamma}$ defined by

$$
\begin{aligned}
\Delta^{-\gamma} f(\boldsymbol{x}) & \stackrel{\text { def }}{=}(2 \pi)^{-d} \\
& \times \int_{\mathbb{R}^{d}} \mathrm{~d} \boldsymbol{\omega} \frac{\mathrm{e}^{\mathrm{j} \boldsymbol{x}^{\top} \boldsymbol{\omega}}-\sum_{|\boldsymbol{k}| \leq\lfloor 2 \gamma-d / 2\rfloor} \frac{{ }_{\mathrm{j}} \boldsymbol{k}_{\mid} \boldsymbol{x}^{\boldsymbol{k}} \boldsymbol{\omega}^{\boldsymbol{k}}}{\boldsymbol{k} !}}{\|\boldsymbol{\omega}\|^{2 \gamma}} \hat{f}(\boldsymbol{\omega}) .
\end{aligned}
$$

It satisfies

$$
\Delta^{\gamma} \Delta^{-\gamma} f=f
$$

for all $f \in \mathcal{S}$ and is called the right inverse. We can extend $\Delta^{-\gamma}$ to a subset of $\mathcal{S}^{\prime}$ by duality

$$
\left\langle\dot{\Delta}^{-\gamma} f, u\right\rangle \stackrel{\text { def }}{=}\left\langle f, \grave{\Delta}^{-\gamma} u\right\rangle
$$

wherever the r.h.s. is meaningful for all $u \in \mathcal{S}$.

While the above definitions may look arbitrary at first glance, they have intuitive interpretations. For example, supposing $f(\boldsymbol{x})$ to be a well-behaved test function whose moments vanish up to degree $\lfloor 2 \gamma-d / 2\rfloor$, (9) simply corresponds to a shift-invariant inverse (all the terms in the sum will be zero in this case), while (10) defines an inverse with all derivatives up to order $\lfloor 2 \gamma-d / 2\rfloor$ forced to be zero at the origin. This latter property is significant in the characterization of fractional Brownian motion as there, by definition, the process should equal zero at $\boldsymbol{x}=\mathbf{0}$.

It also bears mentioning that, unlike the fractional Laplacian, these inverse operators are in general not shift-invariant when

\footnotetext{
3"Regularization" here stands for a general way of assigning a value to an integral with a singular kernel, in a manner that would be consistent with what one would expect when evaluating the integral for a smooth function that vanishes in a neighborhood of the singularity (and for which the integral can be evaluated).
}

applied to members of $\mathcal{S}$ (they are, however, scale- and rotationinvariant in the previously defined sense).

\section{Polyharmonic Splines AND WaVElets}

\section{A. Splines and Operators}

By differentiating a polynomial spline a sufficient number of times, one procures a sum of Dirac deltas located at the knots. This observation underlies a conceptual framework in which splines are defined as functions that are mapped to a sum of Dirac deltas by some suitably chosen operator U. This approach leads to interesting generalizations: one may for example use fractional derivatives to obtain splines of fractional order [34], [46].

Formally, in this framework, given a shift invariant operator $\mathrm{U}$, we define a lattice U-spline as a function $s(\boldsymbol{x})$ for which

$$
\mathrm{U} s(\boldsymbol{x})=\sum_{\boldsymbol{k} \in \mathbb{Z}^{d}} c[\boldsymbol{k}] \delta(\boldsymbol{x}-\mathrm{Q} \boldsymbol{k})
$$

with $c \in \ell_{\infty}\left(\mathbb{Z}^{d}\right)$ and where the points $\mathrm{Q} \boldsymbol{k}$ belong to a lattice. One may try to solve the equation

$$
\mathrm{U} \varrho(\boldsymbol{x})=\delta(\boldsymbol{x})
$$

for $\varrho(\boldsymbol{x})$ (Green's function) by finding an inverse operator. $s(\boldsymbol{x})$ can then be expressed in terms of $\varrho(\boldsymbol{x})$ and its lattice shifts, plus a term from the null-space of $U$; that is

$$
s(\boldsymbol{x})=\sum_{\boldsymbol{k} \in \mathbb{Z}^{d}} c[\boldsymbol{k}] \varrho(\boldsymbol{x}-\mathrm{Q} \boldsymbol{k})+s_{0}(\boldsymbol{x})
$$

with $\mathrm{U} s_{0}=0$.

In practice, it is often of interest to limit oneself to splines $s(\boldsymbol{x}) \in \mathrm{L}_{2}\left(\mathbb{R}^{d}\right)$, in which case we consider a modified version of the above problem, where we introduce a localization operator (filter) $\mathrm{V}_{\mathrm{Q}}$ and study the equation

$$
\mathrm{U} \phi(\boldsymbol{x})=\mathrm{V}_{\mathrm{Q}} \delta(\boldsymbol{x})=\sum_{\boldsymbol{k} \in \mathbb{Z}^{d}} v[k] \delta(\boldsymbol{x}-\mathrm{Q} \boldsymbol{k})
$$

in place of (12). B-splines, which form spatially localized bases for square-integrable spline spaces, are in fact solutions to such equations [34], [37].

In the remainder of this section, we first introduce such localized (B-spline) bases for spaces of square-integrable polyharmonic splines, for which the operator $\mathrm{U}$ is a fractional Laplacian, and $\mathrm{V}_{\mathrm{Q}}$ is its discretization over any one of the lattices introduced in Remark 1. Next, in Section IV-C, we show how these B-splines can act as scaling functions for a multiresolution analysis (Theorem 2). We follow this by the investigation of one of the main properties of wavelets derived from these B-splines, namely that polyharmonic wavelet kernels behave like low-frequency approximations of the fractional Laplacian (Theorem 3).

\section{B. Polyharmonic B-Splines}

If we take the operator $\mathrm{U}$ of the previous subsection to be the fractional Laplacian $\Delta^{\gamma}$, solutions to (11) (which in this case is a polyharmonic equation) are called polyharmonic splines [56]. 
As noted in Section III-B, when the function $\hat{f}(\boldsymbol{\omega})$ has sufficiently many zeros at the origin, the fractional Laplacian can be inverted via (8) without difficulty. Indeed, one of the ways to deal with singular integrals is to multiply the integration kernel by a function that vanishes at the singularity.

It is, therefore, reasonable in our problem to first choose an appropriate localization filter $\mathrm{V}_{\mathrm{Q}}$ whose Fourier symbol $\hat{\mathrm{V}}_{\mathrm{Q}}(\boldsymbol{\omega})$ approximates that of $\Delta^{\gamma}$ at its zero at the origin, thus cancelling the singularity of $\Delta^{-\gamma}$ and permitting us to solve the spline equation

$$
\Delta^{\gamma} \phi_{2 \gamma}(x)=\mathrm{V}_{\mathrm{Q}} \delta(x)
$$

in the Fourier domain, for the B-spline $\phi_{2 \gamma}(\boldsymbol{x})$. Different choices of such an operator $V_{Q}$ lead to different families of polyharmonic B-splines (quasi-isotropic, orthogonal, etc.) [37].

In the simplest case, the elementary localization filter $V_{Q}$ corresponds (up to a factor of $(-1)^{\gamma}$ ) to the $\gamma$ th fractional iteration of an elementary discretization of the Laplacian. Specifically, for $\gamma=1$ we define the elementary localization operator, $\Delta_{Q}$, in the spatial domain as follows.

$$
\Delta_{Q} f \stackrel{\text { def }}{=} \frac{1}{\mu^{2}} \sum_{1 \leq i \leq N} 2 f(\cdot)-f\left(\cdot-\mathrm{Q} \boldsymbol{y}_{i}\right)-f\left(\cdot+\mathrm{Q} \boldsymbol{y}_{i}\right)
$$

(see LAT-2 for the definition of $\boldsymbol{y}_{i} \mathrm{~s}$ ). Note also its Fourier symbol

$$
\hat{\Delta}_{Q}(\boldsymbol{\omega})=\frac{4}{\mu^{2}} \sum_{1 \leq i \leq N} \sin ^{2}\left(\frac{\boldsymbol{y}_{i}^{\top} Q^{\top} \boldsymbol{\omega}}{2}\right) .
$$

For other values of $\gamma>0$, we simply define

$$
\hat{\Delta}_{Q}^{\gamma}(\boldsymbol{\omega}) \stackrel{\text { def }}{=}\left[\hat{\Delta}_{\mathrm{Q}}(\boldsymbol{\omega})\right]^{\gamma} \text {. }
$$

This choice of the localization operator leads to a fractional generalization of Rabut's elementary $\gamma$-harmonic B-splines, here denoted $\phi_{2 \gamma, \mathrm{el}}$ [35], [37].

More generally, the localization operator $\hat{V}_{Q}$ used in (14) can be any one with a Fourier symbol factorizable as

$$
\hat{\mathrm{V}}_{\mathrm{Q}}(\boldsymbol{\omega})=\hat{\Delta}_{\mathrm{Q}}^{\gamma}(\boldsymbol{\omega}) \hat{\mathrm{T}}_{\mathrm{Q}}^{\gamma}(\boldsymbol{\omega})
$$

where $\hat{\mathrm{T}}_{Q}^{\gamma}(\boldsymbol{\omega}) \stackrel{\text { def }}{=}\left[\hat{\mathrm{T}}_{\mathrm{Q}}(\boldsymbol{\omega})\right]^{\gamma}$ is the continuous Fourier expression of some lattice operator (filter), and is bounded from above and below with a strictly positive lower bound. We shall assume $\hat{\mathrm{T}}_{\mathrm{Q}}(\boldsymbol{\omega})$ to be normalized with $\hat{\mathrm{T}}_{\mathrm{Q}}(\mathbf{0})=1$.

Remark 3: The choice of $\hat{\mathrm{T}}_{Q}^{\gamma}(\boldsymbol{\omega})$, apart from these constraints, is essentially arbitrary in so far as it corresponds to a discrete (lattice) filter, as all such choices lead to the same multiresolution subspaces. However, as will be seen shortly, different choices of $\hat{T}_{Q}$ do lead to different B-spline functions spanning the same spaces, and $\hat{T}_{Q}$ may be specifically selected so as to give these functions a desired correlation structure.

The solution to (14) can now be written explicitly in the Fourier domain as

$$
\hat{\phi}_{2 \gamma}(\boldsymbol{\omega})=\frac{\hat{\mathrm{V}}_{\mathrm{Q}}(\boldsymbol{\omega})}{\|\boldsymbol{\omega}\|^{2 \gamma}}=\hat{\mathrm{T}}_{\mathrm{Q}}^{\gamma}(\boldsymbol{\omega}) \frac{\hat{\Delta}_{Q}^{\gamma}(\boldsymbol{\omega})}{\|\boldsymbol{\omega}\|^{2 \gamma}}=\hat{\mathrm{T}}_{\mathrm{Q}}^{\gamma}(\boldsymbol{\omega}) \hat{\phi}_{2 \gamma, \mathrm{el}}(\boldsymbol{\omega})
$$

where $\hat{\phi}_{2 \gamma, \text { el }}(\boldsymbol{\omega}) \stackrel{\text { def }}{=} \hat{\Delta}_{Q}^{\gamma}(\boldsymbol{\omega}) /\|\boldsymbol{\omega}\|^{2 \gamma}$ is the Fourier transform of the elementary $\gamma$-harmonic B-spline $\phi_{2 \gamma, \text { el }}$ that was mentioned before.

In order for the polyharmonic B-spline function $\phi_{2 \gamma}(\boldsymbol{x})$ thus defined to be square-integrable we need to have

$$
\gamma>\frac{d}{4}
$$

The following proposition summarizes the smoothness and integrability properties of $\phi_{2 \gamma}(\boldsymbol{x})$.

Proposition 1: $\phi_{2 \gamma}$, with $\gamma>d / 4$, belongs to the Sobolev space $\mathcal{H}^{s}$ for any $s<2 \gamma-d / 2$.

Proof: Using the Taylor expansion of $\hat{\Delta}_{Q}^{\gamma}(\omega)$, we can immediately see that $\hat{\phi}_{2 \gamma, \mathrm{el}}(\boldsymbol{\omega})$ tends to 1 as $\|\boldsymbol{\omega}\| \rightarrow 0$

$$
\begin{aligned}
\lim _{\|\boldsymbol{\omega}\| \rightarrow 0} \hat{\phi}_{2 \gamma, \mathrm{el}}(\boldsymbol{\omega}) & =\lim _{\|\boldsymbol{\omega}\| \rightarrow 0}\left|\frac{\sum_{i} \frac{4}{\mu^{2}} \sin ^{2}\left(\frac{\boldsymbol{y}_{i}^{\top} Q^{\top} \boldsymbol{\omega}}{2}\right)}{\|\boldsymbol{\omega}\|^{2}}\right|^{\gamma} \\
& =\lim _{\|\boldsymbol{\omega}\| \rightarrow 0}\left|\frac{\boldsymbol{\omega}^{\top}\left(\sum_{i} \frac{\mathrm{Q} \boldsymbol{y}_{i} \boldsymbol{y}_{i}^{\top} Q^{\top}}{\mu^{2}}\right) \boldsymbol{\omega}}{\boldsymbol{\omega}^{\top} \boldsymbol{\omega}}\right|^{\gamma}=1
\end{aligned}
$$

[cf. (5)]. In addition, both $\hat{\Delta}_{Q}^{\gamma}(\boldsymbol{\omega})$ and $\hat{\mathrm{T}}_{\mathrm{Q}}^{\gamma}(\boldsymbol{\omega})$ are by definition continuous and bounded. What all this means is that $\hat{\phi}_{2 \gamma}(\boldsymbol{\omega})$ is continuous and bounded everywhere and decays like $\|\boldsymbol{\omega}\|^{2 \gamma}$ [cf. (16)]. It then follows from the Fourier-domain definition of the Sobolev space $\mathcal{H}^{s}$ that $\phi_{2 \gamma} \in \mathcal{H}^{s}$ for all $s<2 \gamma-d / 2$.

As was already mentioned, the trivial choice of $\hat{\mathrm{T}}_{Q}(\boldsymbol{\omega}) \equiv 1$ in (16) leads to elementary fractional polyharmonic B-splines. Among other possibilities, one can, e.g., opt for the orthogonal polyharmonic B-spline $\phi_{2 \gamma}^{\perp}(\boldsymbol{x})$. In effect, starting from any localization operator $\mathrm{V}_{\mathrm{Q}}$ and its corresponding B-spline $\phi_{2 \gamma}(\boldsymbol{x})$, one can define the orthogonal localization operator $\mathrm{V}_{Q}^{\perp}$ as

$$
\hat{\mathrm{V}}_{\mathrm{Q}}^{\perp}(\boldsymbol{\omega})=\frac{\hat{\mathrm{V}}_{\mathrm{Q}}(\boldsymbol{\omega})}{\sqrt{\left|\hat{\mathrm{A}}_{\mathrm{Q}}(\boldsymbol{\omega})\right|}}
$$

where we have introduced the autocorrelation filter

$$
\hat{\mathrm{A}}_{\mathrm{Q}}(\boldsymbol{\omega}) \stackrel{\text { def }}{=} \hat{\mathrm{A}}_{\mathrm{Q}}\left\{\phi_{2 \gamma}\right\}(\boldsymbol{\omega})=\sum_{\boldsymbol{k} \in \mathbb{Z}^{d}}\left|\hat{\phi}_{2 \gamma}\left(\boldsymbol{\omega}+2 \pi \mathrm{Q}^{-\mathrm{T}} \boldsymbol{k}\right)\right|^{2}
$$

defined as the lattice Fourier transform of $a[k] \stackrel{\text { def }}{=}\left\langle\phi_{2 \gamma}(\cdot-\right.$ $\left.\mathrm{Q} \boldsymbol{k}), \phi_{2 \gamma}(\cdot)\right\rangle$. Division by the square root of $\hat{\mathrm{A}}_{\mathrm{Q}}\left\{\phi_{2 \gamma}\right\}(\boldsymbol{\omega})$ guarantees that $\left\langle\phi_{2 \gamma}^{\perp}(\cdot-\mathrm{Q} \boldsymbol{k}), \phi_{2 \gamma}^{\perp}(\cdot)\right\rangle=\delta_{\boldsymbol{k}}$. The above orthogonalization depends on the positivity and boundedness of $\hat{\mathrm{A}}_{Q}$. The demonstration of these properties is included in the proof of Theorem 2.

\section{Polyharmonic Multiresolution Analysis}

The following theorem allows us to form a multiresolution analysis based on polyharmonic B-splines (in their different flavors).

Theorem 2: The polyharmonic B-splines defined in (16) have the following properties.

MRA-1. They form a partition of unity. 
MRA-2. They fulfil a two-scale relation of the following form:

$$
\phi_{2 \gamma}\left(\mathrm{D}_{\mathrm{Q}}^{-1} \boldsymbol{x}\right)=\sum_{\boldsymbol{k} \in \mathbb{Z}^{d}} h[\boldsymbol{k}] \phi_{2 \gamma}(\boldsymbol{x}-\mathrm{Q} \boldsymbol{k})
$$

with $h \in \ell_{1}\left(\mathbb{Z}^{d}\right)$.

MRA-3. They generate a Riesz basis for their $\ell_{2}$ span.

Proofs are given in Appendix I.

Properties MRA-1-3 are those necessary to form a Mallattype multiresolution analysis [15], [16], [47]. The basic spline approximation subspace is defined as

$$
\mathcal{V}_{2 \gamma, 0} \stackrel{\text { def }}{=}\left\{\sum_{\boldsymbol{k} \in \mathbb{Z}^{d}} c[\boldsymbol{k}] \phi_{2 \gamma}(\cdot-\mathrm{Q} \boldsymbol{k}) \mid c \in \ell_{2}\left(\mathbb{Z}^{d}\right)\right\} .
$$

More generally, the $n$th level multiresolution spline space is

$$
\mathcal{V}_{2 \gamma, n} \stackrel{\text { def }}{=}\left\{f\left(\mathrm{D}_{\mathrm{Q}}^{-n} \cdot\right) \mid f(\cdot) \in \mathcal{V}_{2 \gamma, 0}\right\}
$$

Note that because $\hat{T}_{Q}^{\gamma}(\boldsymbol{\omega})$ is bounded away from zero, the definition of the above spaces is independent of its particular choice. As a consequence of Theorem 2, these spaces are nested

$$
\{0\} \subset \cdots \subset \mathcal{V}_{2 \gamma, 1} \subset \mathcal{V}_{2 \gamma, 0} \subset \mathcal{V}_{2 \gamma,-1} \subset \cdots \subset \mathrm{L}_{2}
$$

and the closure of their union is $\mathrm{L}_{2}$.

The next result concerns the fractional derivatives and integrals of polyharmonic splines, which are polyharmonic splines in their own right, but of a different order (see Appendix I for the proof).

\section{Proposition 2:}

1. The $\gamma_{0}$ th fractional Laplacian of a polyharmonic spline of order $2 \gamma$ belonging to $\mathcal{V}_{2 \gamma, 0}$, with $\gamma>\gamma_{0}$, is a lower order spline in $\mathcal{V}_{2\left(\gamma-\gamma_{0}\right), 0}$.

2. If $\Delta^{\gamma_{0}} s(\boldsymbol{x})$ is a polyharmonic spline of order $2 \gamma$, then $s(\boldsymbol{x})$ is a polyharmonic spline of order $2 \gamma+2 \gamma_{0}$.

Polyharmonic Wavelets: Polyharmonic wavelets can be defined as basis functions that span the orthogonal complements in the series of nested approximation spaces. For a given multiresolution hierarchy, there will in general be $|D|-1$ distinct mother-wavelets $\psi_{2 \gamma}^{i}, 1 \leq i<|\mathrm{D}|$ (we shall subsequently drop the index $i$ as all arguments apply equally to all wavelets).

The semi-orthogonality condition imposed on the wavelet spaces forces the wavelets to have a behavior similar to the operator $\Delta^{\gamma}$ at low frequencies. This quality is encapsulated in the next theorem (a proof is given in Appendix I).

Theorem 3: A semi-orthogonal polyharmonic wavelet of order $2 \gamma$ can be written as

$$
\psi_{2 \gamma}(x)=\Delta^{\gamma} \eta(x)
$$

where $\eta(\boldsymbol{x})$ (the smoothing kernel) is a polyharmonic spline of order $4 \gamma$ that belongs to the Sobolev space $\mathcal{H}^{s}$ for any $s<$ $4 \gamma-d / 2$.

A special case of the general multiresolution construction studied in this section can be found in a previous paper [37], where an explicit construction scheme for the 2-D quincunx lattice (requiring the design of only one mother wavelet) was provided.

\section{CHARACTERIZATION OF FRACTIONAL BROWNIAN RANDOM FIELDS}

A random field is said to be self-similar when applying a similarity transform to its domain does not change its stochastic behavior (apart from a possible renormalization factor). For a review of self-similar random fractals we refer the reader to Benassi and Istas [32]. Gaussian self-similar processes were also studied by Dobrushin in his 1979 paper [48].

Fractional Brownian motions form a subset of (continuous) self-similar fields distinguished by their Gaussian statistics and stationary increments [3]. Stochastic self-similarity and stationary increments in particular force the fields to have homogeneous (self-similar) variance functions. Given that fBms are Gaussian and, hence, are fully defined by their second-order statistics, one traditional way of characterizing them is by specifying their variogram, which, for a normalized $\mathrm{fBm}$ of Hurst exponent $H$, has the following form [49, ch. 18]:

$$
\mathbf{E}\left\{\left|\mathfrak{B}_{H}(\boldsymbol{x})-\mathfrak{B}_{H}\left(\boldsymbol{x}^{\prime}\right)\right|^{2}\right\}=2\left\|\boldsymbol{x}-\boldsymbol{x}^{\prime}\right\|^{2 H} .
$$

$\mathfrak{B}_{H}$ is additionally postulated to have zero mean and to be zero at $x=\mathbf{0}$ almost surely. One remarks that the derived variance function is indeed homogeneous

$$
\mathbf{E}\left\{\left|\mathfrak{B}_{H}(\boldsymbol{x})\right|^{2}\right\}=2\|\boldsymbol{x}\|^{2 H} .
$$

Some of the other definitions of $\mathrm{fBm}$ fields are in terms of integrals of white noise [50] and by their spectral harmonizable representation [31], [51]. (The latter formulation is closely related to what we present in the sequel. See Remark 4).

An important approach to characterization often used in the analysis and synthesis of stationary random processes relies on the notion of whitening. In this formulation, an operator is sought after which whitens the process in question, i.e., maps it to white noise. Next, a suitable inverse operator needs to be identified, which can then be applied to white noise in order to recreate instances of the desired random process. While standard in the study of stationary processes, this scheme can be extended to certain nonstationary cases, and in particular to the definition of $\mathrm{fBm}$, by adopting a distribution theoretic formalism. This will be demonstrated in this section.

In effect, in the sequel we will show that fractional Laplacians introduced previously whiten multivariate $\mathrm{fBm}$ fields of corresponding $H$ exponent (as also discussed by Benassi et al. [31]); that is

$$
\Delta^{H / 2+d / 4} \mathfrak{B}_{H}=\epsilon_{H} \mathfrak{W}
$$

where $\mathfrak{W}$ is normalized white Gaussian noise and $\epsilon_{H}$ is a constant. We also show that an fBm field may be obtained by applying the right inverse (cf. Section III-B) to white Gaussian noise, which is to say that

$$
\mathfrak{B}_{H}=\epsilon_{H} \dot{\Delta}^{-H / 2-d / 4} \mathfrak{W} .
$$

In addition to being conceptually interesting, the above characterization of multivariate fractional Brownian motion leads to 


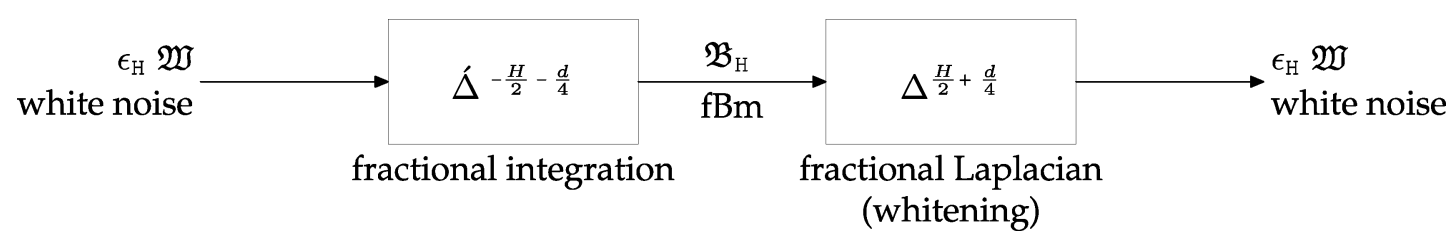

Fig. 1. Innovation model for multivariate fractional Brownian motion.

a natural generalization of the definition to values of $H$ outside the $(0,1)$ range.

Furthermore, fractal properties of the process find their correspondent in the operator: the scale-invariance property imposed on the operator induces the statistical self-similarity of the process, while rotation-invariance entails its statistical isotropy.

These results all follow from a multivariate generalization of Theorem 1 of Blu and Unser [30], which provides a spectral characterization of $\mathrm{fBm}$ through its characteristic functional (cf. Section II-C).

Theorem 4: Let $0<H<1$. An fBm field with Hurst parameter $H$ and variogram $2\left\|\boldsymbol{x}-\boldsymbol{x}^{\prime}\right\|^{2 H}$ has the following characteristic functional:

$$
\mathbf{Z}_{\mathfrak{B}_{H}}(u)=\exp \left(-\frac{\epsilon_{H}^{2}}{2(2 \pi)^{d}} \int \mathrm{d} \boldsymbol{\omega} \frac{|\hat{u}(\boldsymbol{\omega})-\hat{u}(\mathbf{0})|^{2}}{\|\boldsymbol{\omega}\|^{2 H+d}}\right)
$$

where

$$
\epsilon_{H}^{2}=-2^{2 H+d} \pi^{d / 2} \frac{\Gamma\left(H+\frac{d}{2}\right)}{\Gamma(-H)} .
$$

Proof: A complete proof can be found in Appendix II. The main step of the demonstration consists in showing that (20) defines a Gaussian process whose correlation function $c_{\mathfrak{B}_{H}}\left(\boldsymbol{x}, \boldsymbol{x}^{\prime}\right)$ is that of an isotropic fractional Brownian motion with Hurst parameter $H$, that is, the function

$$
c_{\mathfrak{B}_{H}}\left(\boldsymbol{x}, \boldsymbol{x}^{\prime}\right)=\|\boldsymbol{x}\|^{2 H}+\left\|\boldsymbol{x}^{\prime}\right\|^{2 H}-\left\|\boldsymbol{x}-\boldsymbol{x}^{\prime}\right\|^{2 H} .
$$

We recall the characteristic functional of the unit random field $\mathfrak{W}$ (a.k.a. white Gaussian noise)

$$
\begin{aligned}
\mathbf{Z}_{\mathfrak{W}}(u) & =\exp \left(-\frac{1}{2} \int \mathrm{d} \boldsymbol{x}|u(\boldsymbol{x})|^{2}\right) \\
& =\exp \left(-\frac{1}{2}(2 \pi)^{-d} \int \mathrm{d} \boldsymbol{\omega}|\hat{u}(\boldsymbol{\omega})|^{2}\right) .
\end{aligned}
$$

From comparing this with (20) and by applying a duality argument, we can deduce that

$$
\mathbf{Z}_{\mathfrak{B}_{H}}(u)=\mathbf{Z}_{\mathfrak{W}}\left(\epsilon_{H} \grave{\Delta}^{-\gamma_{0}} u\right)=\mathbf{Z}_{\epsilon_{H} \dot{\Delta}^{-\gamma_{0} \mathfrak{W}}}(u)
$$

with $\gamma_{0}=H / 2+d / 4$. This means that the random field obtained by applying the right inverse $\Delta^{-\gamma_{0}}$ to the unit (generalized) random field $\mathfrak{W}$ is a multivariate $\mathrm{fBm}$ with Hurst parameter $H$, i.e.,

$$
\mathfrak{B}_{H}=\epsilon_{H} \dot{\Delta}^{-H / 2-d / 4} \mathfrak{W} \text {. }
$$

Equation (22) is an alternative characterization of fractional Brownian motion, and can be used to extend the definition to noninteger $H>1$. The covariance function of these extensions can be obtained with the aid of Lemma 1 of Appendix II.

It also follows that fractional Brownian motion is whitened by the fractional Laplacian operator

$$
\Delta^{H / 2+d / 4} \mathfrak{B}_{H}=\epsilon_{H} \mathfrak{W}
$$

a fact that leads to the innovation model depicted in Fig. 1.

Remark 4: For $0<H<1$, a related characterization of real fractional Brownian fields is by their harmonizable representation as the stochastic integral

$$
\int_{\mathbb{R}^{d}} \frac{\mathrm{e}^{\mathrm{j} \boldsymbol{x}^{\top} \boldsymbol{\omega}}-1}{\|\boldsymbol{\omega}\|^{H+d / 2}} \widehat{W}(\mathrm{~d} \omega)
$$

where $\widehat{W}$ is a (Hermitian symmetric) complex random measure corresponding to the Fourier transform of real-valued white Gaussian noise (see Samorodnitsky and Taqqu [51] for an in-depth discussion of the single-parameter case). The integrand $\left(\mathrm{e}^{\mathrm{j} \boldsymbol{x}^{\top} \boldsymbol{\omega}}-1\right) /\|\boldsymbol{\omega}\|^{H+d / 2}$ is comparable to the spectral representation of the right inverse in (10), which reduces to the said integrand for $0<H<1$. The treatment of Benassi et al. [31] is also of direct pertinency, and includes similar extensions.

\section{Polyharmonic WaVelet ANALysis of MULTIVARIATE FBM}

Considering the inherent link between polyharmonic splines and $\mathrm{fBms}$ that has been emphasized throughout this article, it should not come as a surprise that a wavelet analysis of multivariate $\mathrm{fBm}$ would have interesting properties. We study some of these in the first part of this section. Next, we complement and verify our derivations through some experimental results.

\section{A. Probability Distribution of Wavelet Coefficients}

Proposition 3: The polyharmonic spline wavelet transform of order $2 \gamma \geq 2 \gamma_{0}$, with $\gamma_{0} \stackrel{\text { def }}{=} H / 2+d / 4$, maps the nonstationary process $\mathfrak{B}_{H}$ into a series of stationary (discrete) Gaussian processes.

Proof: We can rely on Theorem 3 and the innovation model to see that, e.g., the wavelet coefficients at level $n=0$ are stationary Gaussian processes obtained by filtering white noise

$$
\begin{aligned}
w_{0}[\boldsymbol{k}] & =\left\langle\mathfrak{B}_{H}, \psi_{2 \gamma}(\cdot-\mathrm{Q} \boldsymbol{k})\right\rangle=\left\langle\Delta^{\gamma_{0}} \mathfrak{B}_{H}, \Delta^{\gamma-\gamma_{0}} \eta(\cdot-\mathrm{Q} \boldsymbol{k})\right\rangle \\
& =\left\langle\epsilon_{H} \mathfrak{W}, \Delta^{\gamma-\gamma_{0}} \eta(\cdot-\mathrm{Q} \boldsymbol{k})\right\rangle .
\end{aligned}
$$

(Note that even though the polyharmonic spline $\Delta^{\gamma-\gamma_{0}} \eta(\cdot-\mathrm{Q} k$ ) is not a Schwartz test function, its inner-product with the white 
noise process is nonetheless well-defined as it is continuous and belongs to $\mathcal{H}^{s}$ for some $s>0$; cf. Theorem 3 ). The demonstration for an arbitrary level $n$ is similar, except that a scale-dependent normalization factor also appears.

What this property means is that the $w_{0}[\boldsymbol{k}] \mathrm{s}$ correspond to the lattice samples of a stationary process with power spectrum $\left.\epsilon_{H}^{2}\|\boldsymbol{\omega}\|\right|^{2 \gamma-2 \gamma_{0}}|\hat{\eta}(\boldsymbol{\omega})|^{2}$ (which is well-defined in the $\mathrm{L}_{2}$ sense since $\eta \in \mathcal{H}^{s}$ for all $\left.s<4 \gamma-d / 2\right)$. This relation is essentially scale-invariant up to a proportionality factor.

Proposition 4: The variance of the polyharmonic wavelet coefficients depends exponentially on the Hurst exponent and the scale $n$ as per

$$
\mathbf{E}\left\{w_{n}^{2}[\boldsymbol{k}]\right\}=|\mathrm{D}|^{(2 H+d) n / d} \mathbf{E}\left\{w_{0}^{2}[\boldsymbol{k}]\right\} .
$$

Proof: This property can be shown using the correlation form $\left\langle\langle\cdot, \cdot\rangle_{\mathfrak{B}_{H}}\right.$. One has [cf. (28)]

$$
\begin{aligned}
& \mathbf{E}\left\{w_{n}^{2}[\boldsymbol{k}]\right\} \\
& =\left\langle\left\langle|\mathrm{D}|^{-n / 2} \psi_{2 \gamma}\left(\mathrm{D}_{\mathrm{Q}}^{-n} \boldsymbol{x}-\mathrm{Q} \boldsymbol{k}\right),|\mathrm{D}|^{-n / 2} \psi_{2 \gamma}\left(\mathrm{D}_{\mathrm{Q}}^{-n} \boldsymbol{x}-\mathrm{Q} \boldsymbol{k}\right)\right\rangle_{\mathfrak{B}_{H}}\right. \\
& =\frac{\epsilon_{H}^{2}}{(2 \pi)^{d}} \int \mathrm{d} \boldsymbol{\omega} \frac{\left|\hat{\psi}_{2 \gamma}(\boldsymbol{\omega})\right|^{2}}{\left\|\mathrm{Q}^{-\mathrm{T}} \mathrm{D}^{-n \top} \mathrm{Q}^{\top} \boldsymbol{\omega}\right\|^{2 H+d}} \\
& =|\mathrm{D}|^{(n / d)(2 H+d)} \frac{\epsilon_{H}^{2}}{(2 \pi)^{d}} \int \mathrm{d} \boldsymbol{\omega} \frac{\left|\hat{\psi}_{2 \gamma}(\boldsymbol{\omega})\right|^{2}}{\|\boldsymbol{\omega}\|^{2 H+d}} \\
& =|\mathrm{D}|^{(n / d)(2 H+d)}\left\langle\left\langle\psi_{2 \gamma}(\boldsymbol{x}), \psi_{2 \gamma}(\boldsymbol{x})\right\rangle_{\mathfrak{B}_{H}}\right. \\
& =|\mathrm{D}|^{(n / d)(2 H+d)} \mathbf{E}\left\{w_{0}^{2}[k]\right\} .
\end{aligned}
$$

More generally, we have the following result.

Proposition 5: The covariance of intrascale wavelet coefficients is given by the relation

$$
\begin{aligned}
& \frac{\mathbf{E}\left\{w_{n}[\boldsymbol{k}] w_{n}\left[\boldsymbol{k}^{\prime}\right]\right\}}{|\mathrm{D}|^{(n / d)(2 H+d)}}
\end{aligned}
$$

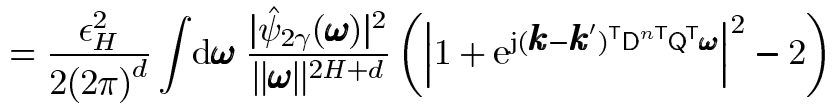

$$
\begin{aligned}
& =\mathbf{E}\left\{w_{0}\left[\mathrm{D}^{n} \boldsymbol{k}\right] w_{0}\left[\mathrm{D}^{n} \boldsymbol{k}^{\prime}\right]\right\} \text {. }
\end{aligned}
$$

Proof: At scale 0 we have

$$
\begin{array}{r}
\mathbf{E}\left\{w_{0}[\boldsymbol{k}] w_{0}\left[\boldsymbol{k}^{\prime}\right]\right\}=\frac{1}{2}\left[\left\langle\psi_{2 \gamma, \boldsymbol{k}}+\psi_{2 \gamma, \boldsymbol{k}^{\prime}}, \psi_{2 \gamma, \boldsymbol{k}}+\psi_{2 \gamma, \boldsymbol{k}^{\prime}}\right\rangle\right\rangle_{\mathfrak{B}_{H}} \\
\left.-\left\langle\left\langle\psi_{2 \gamma, \boldsymbol{k}}, \psi_{2 \gamma, \boldsymbol{k}}\right\rangle\right\rangle_{\mathfrak{B}_{H}}-\left\langle\left\langle\psi_{2 \gamma, \boldsymbol{k}^{\prime}}, \psi_{2 \gamma, \boldsymbol{k}^{\prime}}\right\rangle\right\rangle_{\mathfrak{B}_{H}}\right] .
\end{array}
$$

The proposition is then proved using (28) and with a change of variables as in the previous proof.

Remark 5: The above result can be compared with those obtained by Meyer et al. [23] in the 1-D setting. The wavelets proposed by Meyer $e t$ al. depend on the Hurst parameter $H$ that is matched to the Hurst exponent of the 1-D fBm process in consideration (which should be known a priori). Independence of the wavelet coefficients (true whitening) is a consequence of this perfect match. This in fact corresponds to the wavelets being orthogonal in terms of the positive-definite form $\left\langle\langle\cdot, \cdot\rangle_{\mathfrak{B}_{H}}\right.$. Since this design depends on the Hurst exponent being known, in the problem of estimating $H$ a parameter higher than the true unknown value must be used, in which case the wavelet coefficients will again be correlated. Also note that the results pro- vided in the present paper are general and concern any family of semi-orthogonal polyharmonic wavelets. In the actual implementation of wavelets for a given lattice, there is some room for incorporating certain desired behaviors in the design of the wavelet filter, which will in turn affect the smoothing function of Theorem 3.

As a demonstration of potential, the above results (Propositions 3 and 4 in particular) allow us to extend 1-D wavelet estimators of the Hurst exponent reported in the literature [18], [20], [27]-[29] to the multidimensional setting. In its simplest form, estimation can be based on the identity

$$
\log _{\sqrt[d]{|| \mathbb{|} \mid}}\left(\mathbf{E}\left\{w_{n}^{2}[k]\right\}\right)=(2 H+d) n+C
$$

where $C=\log _{\sqrt[d]{|D|}}\left(\boldsymbol{E}\left\{w_{0}^{2}[\boldsymbol{k}]\right\}\right)$ is a computable constant that depends on the choice of the wavelet (Proposition 4). This means that a linear regression of the estimates of the variance in each sub-band in the log scale provides an estimate of $H$.

An improved estimate may be obtained using a maximumlikelihood (ML) formulation. This is essentially a multidimensional adaptation of the ML-estimator of Wornell [27], [29]. ${ }^{4}$ The estimate is defined as the minimizer of a negative log-likelihood approximate (leaving out the constant term)

$$
\ell(\boldsymbol{w} \mid \boldsymbol{\theta})=\frac{1}{2} \sum_{n \in \mathcal{N}} N_{n} \log \sigma_{n}^{2}(\boldsymbol{\theta})+\frac{E_{n}}{\sigma_{n}^{2}(\boldsymbol{\theta})} .
$$

In the above formula $\boldsymbol{\theta} \stackrel{\text { def }}{=}\left(H, C^{\prime}\right)$-with $C^{\prime}$ a normalization factor - is the set of parameters to estimate; $\mathcal{N}$ is the set of levels used for estimation; $N_{n}$ denotes the number of coefficients at level $n$

$$
\sigma_{n}^{2}(\boldsymbol{\theta}) \stackrel{\text { def }}{=} \mathbf{E}\left\{w_{n}^{2}[\boldsymbol{k}]\right\}=C^{\prime}|\mathrm{D}|^{(2 H+d) n / d}
$$

is the theoretical variance of level $n$ wavelet coefficients (cf. Proposition 4); and, finally, $E_{n}$ is the observed wavelet energy (i.e., the sum of coefficients squared) at level $n$. In the implementation we have used the previous regression estimate as an initial guess and applied Newton's method to the derivative of $\ell$. This provides a fast (essentially real-time) way of producing an improved estimate of $H$.

MATLAB implementations of the above estimators are available via our website (http://bigwww.epfl.ch/demo/fractaldimension/).

\section{B. Experimental Results}

The estimation procedure outlined previously was applied to instances of (periodic, due to discretization) 2-D fBm, generated via Fourier domain filtering as per Section III-B [cf. (22) and Remark 4]. The wavelets used for analysis were isotropic polyharmonic wavelets of Van De Ville et al. [37], which have a fast FFT-based implementation. The order of the wavelets was chosen to exceed $H+d / 2$ in order to satisfy the requirements of Proposition 3. We used a quincunx subsampling scheme, which

\footnotetext{
${ }^{4}$ Note that, as is the case for the cited estimators, the ML formulation is approximate where the wavelet is not specifically designed to exactly match the process, as the correlation between wavelet coefficients is not taken into consideration. We have provided formulae for the covariances, which could in principle be used to improve the estimate. This, however, would substantially complicate the estimator.
} 


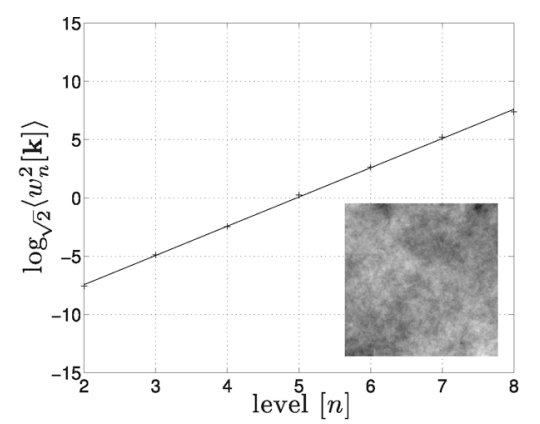

(a) $\mathrm{fBm}\left(H=0.30, \hat{H}_{\mathrm{log}}=0.25, \hat{H}_{\mathrm{ML}}=0.28\right)$

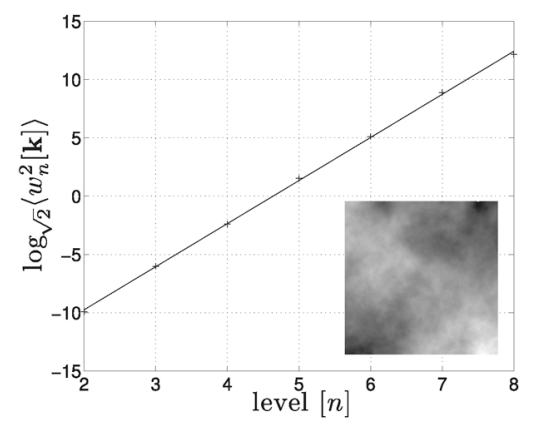

(c) $\operatorname{fBm}\left(H=0.90, \hat{H}_{\log }=0.85, \hat{H}_{\mathrm{ML}}=0.88\right)$

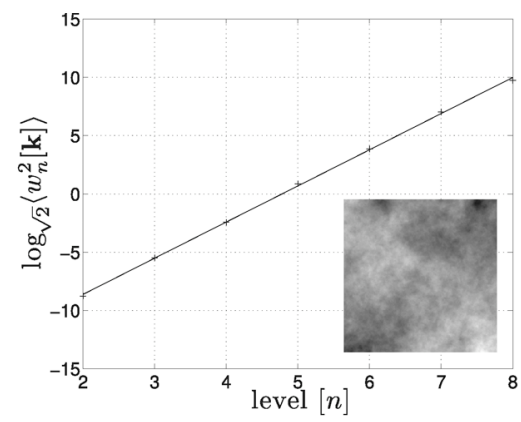

(b) $\mathrm{fBm}\left(H=0.60, \hat{H}_{\log }=0.55, \hat{H}_{\mathrm{ML}}=0.58\right)$

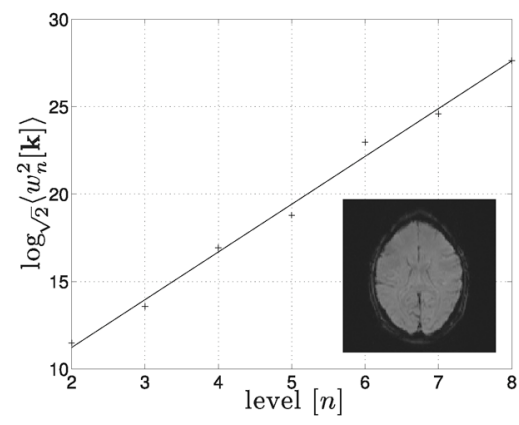

(d) fMRI ( $\left.\hat{H}_{\log }=0.37, \hat{H}_{\mathrm{ML}}=0.34\right)$

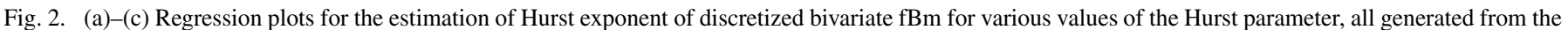
same instance of pseudo-random noise; (d) regression plot for an fMRI image (original images are given as insets).

TABLE I

WAVELET-BASED ESTIMATION OF $H$ (100 REALIZATIONS)

\begin{tabular}{|c|cc|cc|}
\hline \hline true value & \multicolumn{2}{|c|}{ log-regression estimate } & \multicolumn{2}{c|}{ ML estimate } \\
& mean & stdev & mean & stdev \\
\hline 0.3 & 0.290 & 0.007 & 0.293 & 0.004 \\
0.6 & 0.590 & 0.008 & 0.593 & 0.004 \\
0.9 & 0.890 & 0.008 & 0.893 & 0.005 \\
\hline
\end{tabular}

offers a more gradual scale progression, thus furnishing more regression points for the estimation. Another advantage is that the quincunx design involves only a single mother-wavelet.

Hurst parameter estimation was performed on 100 instances of $512 \times 512 \mathrm{fBm}$ images for three different values of $H(0.3$, 0.6, and 0.9). Decomposition levels 2 to 8 were used for estimation. Examples of $\mathrm{fBm}$ images and corresponding regression curves can be seen in Fig. 2. The average and standard deviation of the estimated values, obtained by regression and ML estimation are given in Table I. In experiments we noticed very good fits and small standard deviations, which underline the robustness of the process.

Results of the same analysis applied to a single axial slice of a functional magnetic resonance image (fMRI) of the brain are also shown in Fig. 2. Boundary and background wavelet coefficients were discarded for the analysis in order to avoid boundary effects. The corresponding fractal dimension according to the improved estimate is $d+1-H=2.66$.

It has been suggested that anatomical growth processes lead to fractal-like structures. In the case of the brain, Bullmore et al. [52] have argued that the boundary between the white matter and the cerebral cortex has a fractal-like shape. Additionally, based on recently made possible 3-D high-resolution imaging of the vasculature [53], the branching of the tree structure of the arteries appears to constitute a fractal organization in space. As fMR imaging of brain tissue indirectly measures the flow of oxygenated blood, these arguments can in a way account for the fractal behavior evidenced in Fig. 2(d).

\section{CONCLUSION}

Our approach in this paper was based on the observation that certain families of splines and random processes can be characterized as deterministic vs stochastic solutions of the same fractional partial differential equation.

Motivated by the works of Duchon [43], Arigovindan [44], and Kybic et al. [42] on invariances, in this paper we focused on a particular class of such equations that is singled out by imposing certain fundamental invariance properties on the operator involved. This pointed us to a family of fractional differential operators that are invariant to the translation, rotation, and scaling of the coordinate system. We substantiated the following points.

- These operators (which turn out to be fractional iterations of the Laplacian) lead naturally to the definition of polyharmonic B-splines and multiresolution spline spaces over a large family of multidimensional lattices.

- The same operators whiten multivariate fractional Brownian motion, and can thus be used to rigorously characterize this important family of random fields.

- The relation between deterministic and stochastic formulations provides a natural framework for the analysis of $\mathrm{fBm}$. In particular, a polyharmonic multiresolution analysis of fractional Brownian motion has interesting properties that 
can be deduced from the parallelism between the two formulations. As an example, we showed an application of this observtion in the estimation of the Hurst parameter associated with $\mathrm{fBm}$ processes.

Our results relate, generalize, and formalize previous results of multiple authors, including those of Rabut et al. [35], [36] and Van De Ville et al. [37] (on polyharmonic splines and wavelets), Blu and Unser [30], [34] (on the distributional characterization of 1-D fBm), and Flandrin, Wornell, and Veitch and Abry [20], [27], [28] (on the wavelet analysis of 1-D fBm). In addition, given the generality of the approach, it opens an interesting avenue of research for the future investigation of any of these subjects.

\section{APPENDIX I}

\section{PROOFS OF THEOREMS 2 AND 3 AND OF PROPOSITION 2}

\section{Proof of Theorem 2:}

Proof of MRA-1: By (16), the zeros of $\hat{\phi}_{2 \gamma}(\boldsymbol{\omega})$ are the same as those of $\hat{\Delta}_{Q}^{\gamma}(\boldsymbol{\omega})$, with the exception of the zero at $\boldsymbol{\omega}=\mathbf{0}$ which disappears (see the proof of Proposition 1). From (15) we can see that $\hat{\Delta}_{Q}^{\gamma}(\boldsymbol{\omega})$ is zero iff

$$
\boldsymbol{y}_{i}^{\top} Q^{\top} \frac{\omega}{2 \pi} \in \mathbb{Z} \quad \text { for all } i \text {. }
$$

Since the vectors $\mathrm{Q} \boldsymbol{y}_{i}$ generate $£_{0}$, by the definition of the dual lattice, the above condition is equivalent to

$$
\frac{\boldsymbol{\omega}}{2 \pi} \in £_{0}^{*} .
$$

Removing the zero at $\boldsymbol{w}=\mathbf{0}$ produces $2 \pi £_{0}^{*} \backslash\{\mathbf{0}\}$ as the set of zeros of $\hat{\phi}_{2 \gamma}(\boldsymbol{\omega})$.

Property MRA-1 is then a consequence of the Poisson summation formula [cf. (4); also of direct relevance is Kolountzakis [54, Eqn (5)]].

Proof of MRA-2: Property MRA-2 can be verified by writing the Fourier expression of the refinement filter $h$ as

$$
\hat{\mathrm{H}}_{\mathrm{Q}}(\boldsymbol{\omega})=|\mathrm{D}| \frac{\hat{\phi}_{2 \gamma}\left(\mathrm{D}_{\mathrm{Q}}^{*} \boldsymbol{\omega}\right)}{\hat{\phi}_{2 \gamma}(\boldsymbol{\omega})}=|\mathrm{D}| \frac{\frac{\hat{\mathrm{V}}_{\mathrm{Q}}\left(\mathrm{D}_{\mathrm{Q}}^{*} \boldsymbol{\omega}\right)}{\left\|\mathrm{D}_{\mathrm{Q}}^{*} \boldsymbol{\omega}\right\|^{2 \gamma}}}{\frac{\hat{\mathrm{V}}_{Q}(\boldsymbol{\omega})}{\|\boldsymbol{\omega}\|^{2 \gamma}}}=|\mathrm{D}|^{1-2 \gamma / d} \frac{\hat{\mathrm{V}}_{\mathrm{Q}}\left(\mathrm{D}_{\mathrm{Q}}^{*} \boldsymbol{\omega}\right)}{\hat{\mathrm{V}}_{\mathrm{Q}}(\boldsymbol{\omega})} \text {. }
$$

(The last step results from $D_{Q}^{*}$ being, per definition, a similarity transform matrix; cf. LAT-1). We observe that (i) the numerator and denominator of the last expression are, respectively, $2 \pi £_{-1}^{*}$ - and $2 \pi £_{0}^{*}$-periodic; that (ii) the zeros of the numerator and the denominator happen respectively over the sets $2 \pi £_{-1}^{*}$ and $2 \pi £_{0}^{*}$ and are all of order $2 \gamma$; and finally, that (iii) both the numerator and the denominator are bounded.

We know from (3) that $2 \pi £_{0}^{*} \subset 2 \pi £_{-1}^{*}$. Therefore, first, from (i) it follows that $\hat{H}_{Q}(\boldsymbol{\omega})$ is $2 \pi £_{0}^{*}$-periodic. Secondly, from (ii) and (iii) one concludes that $\hat{H}_{Q}(\boldsymbol{\omega})$ is bounded, with its set of zeros being

$$
\begin{aligned}
\left\{\boldsymbol{\omega} \mid \hat{\mathrm{H}}_{\mathrm{Q}}(\boldsymbol{\omega})=0\right\} & =2 \pi\left(£_{-1}^{*} \backslash £_{0}^{*}\right) \\
& =2 \pi \bigcup_{1 \leq i<|\mathrm{D}|}\left(£_{0}^{*}+\mathrm{Q}^{-\mathrm{T}} \mathrm{D}^{-\mathrm{T}} \boldsymbol{\zeta}_{i}^{*}\right) .
\end{aligned}
$$

These observations establish that $\hat{\mathrm{H}}_{Q}(\boldsymbol{\omega})$ is the lattice Fourier transform of a sequence $h \in \ell_{1}$. The two-scale relation therefore holds.
Proof of MRA-3: Proving the existence of lower and upper Riesz bounds is equivalent to showing that the Fourier transform of the autocorrelation filter (18) is bounded away from zero.

Since $\hat{\mathrm{A}}_{Q}(\boldsymbol{\omega})$ is $2 \pi £_{0}^{*}$-periodic, we can restrict our attention to the unit cell corresponding to the Voronoi region of $\mathbf{0}$ with respect to $2 \pi £_{0}^{*}$. Within this region, we rewrite (18), replacing $\hat{\phi}_{2 \gamma}$ from (16) and noting the periodicity and boundedness of $\hat{\mathrm{V}}_{Q}(\boldsymbol{\omega})$ to obtain

$$
\begin{aligned}
\hat{\mathrm{A}}_{\mathrm{Q}}(\boldsymbol{\omega}) & =\sum_{\boldsymbol{k} \in \mathbb{Z}^{d}} \frac{\hat{\mathrm{V}}_{\mathrm{Q}, 4 \gamma}(\boldsymbol{\omega})}{\left|\boldsymbol{\omega}+2 \pi \mathrm{Q}^{-\mathrm{T}} \boldsymbol{k}\right|^{4 \gamma}} \\
& =\frac{\hat{\mathrm{V}}_{\mathrm{Q}, 4 \gamma}(\boldsymbol{\omega})}{|\boldsymbol{\omega}|^{4 \gamma}}+\hat{\mathrm{V}}_{\mathrm{Q}, 4 \gamma}(\boldsymbol{\omega}) \sum_{\boldsymbol{k} \in \mathbb{Z}^{d} \backslash\{\mathbf{0}\}}\left|\boldsymbol{\omega}+2 \pi \mathrm{Q}^{-\mathrm{T}} \boldsymbol{k}\right|^{-4 \gamma} .
\end{aligned}
$$

The existence of a positive lower bound is then evident as $\hat{\mathrm{A}}_{\mathrm{Q}}(\boldsymbol{\omega})$ is bounded from below by $\hat{\phi}_{4 \gamma}(\boldsymbol{\omega})=\hat{\mathrm{V}}_{\mathrm{Q}, 4 \gamma}(\boldsymbol{\omega}) /|\boldsymbol{\omega}|^{4 \gamma}$, which is strictly positive in the noted region.

Also, since we assumed $\gamma>d / 4$, the second sum converges for all $\boldsymbol{\omega}$ in the unit cell, and is bounded from above (with both factors being bounded). This, in addition to the boundedness of $\hat{\phi}_{4 \gamma}(\boldsymbol{\omega})$, confirms the existence of an upper bound and completes the proof of the Riesz property.

Proof of Proposition 2:

Proof of 1: Any element $f(x)$ of $\mathcal{V}_{2 \gamma, 0}$ can be expressed in the Fourier domain as

$$
\hat{\mathrm{C}}_{\mathrm{Q}}(\boldsymbol{\omega}) \frac{\hat{\Delta}_{\mathrm{Q}}^{\gamma}(\boldsymbol{\omega})}{\|\boldsymbol{\omega}\|^{2 \gamma}}
$$

where the $2 \pi £_{0}^{*}$-periodic and locally square integrable function $\hat{\mathrm{C}}_{Q}(\boldsymbol{\omega})$ is the lattice Fourier transform of a sequence $c \in \ell_{2}$. By applying $\Delta^{\gamma_{0}}$ to $f$ we shall have

$$
\begin{aligned}
\mathcal{F}\left\{\Delta^{\gamma_{0}} f\right\} & =\hat{\mathrm{C}}_{\mathrm{Q}}(\boldsymbol{\omega}) \hat{\Delta}_{\mathrm{Q}}^{\gamma_{0}}(\boldsymbol{\omega}) \frac{\hat{\Delta}_{\mathrm{Q}}^{\left(\gamma-\gamma_{0}\right)}(\boldsymbol{\omega})}{\|\boldsymbol{\omega}\|^{2\left(\gamma-\gamma_{0}\right)}} \\
& =\hat{\mathrm{C}}_{\mathrm{Q}}(\boldsymbol{\omega}) \hat{\Delta}_{Q}^{\gamma_{0}}(\boldsymbol{\omega}) \hat{\phi}_{2\left(\gamma-\gamma_{0}\right), \mathrm{el}}(\boldsymbol{\omega}) .
\end{aligned}
$$

Since $\hat{\mathrm{C}}_{Q}(\boldsymbol{\omega}) \hat{\Delta}_{Q}^{\gamma_{0}}(\boldsymbol{\omega})$ is also a $2 \pi £_{0}^{*}$-periodic and locally square integrable function (due to the periodicity and boundedness of the second factor), it corresponds to the Fourier transform of some $\ell_{2}$ sequence $c^{\prime}$. $\Delta^{\gamma_{0}} f$ can, therefore, be written in the form

$$
\sum_{\boldsymbol{k} \in \mathbb{Z}^{d}} c^{\prime}[\boldsymbol{k}] \phi_{2\left(\gamma-\gamma_{0}\right)}(\cdot-\mathrm{Q} \boldsymbol{k})
$$

whereby $\Delta^{\gamma_{0}} f \in \mathcal{V}_{\left(\gamma-\gamma_{0}\right), 0}$.

Proof of 2: From the assumption, by the definition of polyharmonic splines [see (11)], we have

$$
\Delta^{\gamma} \Delta^{\gamma_{0}} s(\boldsymbol{x})=\sum_{\boldsymbol{k} \in \mathbb{Z}^{d}} c[\boldsymbol{k}] \delta(\boldsymbol{x}-\mathrm{Q} \boldsymbol{k}) .
$$

Using (7), we can write

$$
\Delta^{\gamma+\gamma_{0}} s(\boldsymbol{x})=\sum_{\boldsymbol{k} \in \mathbb{Z}^{d}} c[\boldsymbol{k}] \delta(\boldsymbol{x}-\mathrm{Q} \boldsymbol{k})
$$

which, per definition, establishes $s(\boldsymbol{x})$ as a polyharmonic spline of order $2 \gamma+2 \gamma_{0}$. 
Proof of Theorem 3:

Proof: The semi-orthogonality condition is equivalent to stating that

$$
\left\langle\phi_{2 \gamma}\left(\mathrm{D}_{\mathrm{Q}}^{-1} \boldsymbol{x}\right), \psi_{2 \gamma}\left(\mathrm{D}_{\mathrm{Q}}^{-1} \boldsymbol{x}-\mathrm{Q} \boldsymbol{k}\right)\right\rangle \equiv 0 .
$$

We replace the B-spline $\phi_{2 \gamma}$ and the wavelet $\psi_{2 \gamma}$ in the above equality by their higher resolution B-spline expansions, given in (19) for $\phi_{2 \gamma}$ and below for $\psi_{2 \gamma}$

$$
\psi_{2 \gamma}\left(\mathrm{D}_{\mathrm{Q}}^{-1} \boldsymbol{x}\right)=\sum_{\boldsymbol{k} \in \mathbb{Z}^{d}} g[\boldsymbol{k}] \phi_{2 \gamma}(\boldsymbol{x}-\mathrm{Q} \boldsymbol{k})
$$

where $g \in \ell_{1}$ is the stable wavelet filter. The autocorrelation filter $a[\boldsymbol{k}] \stackrel{\text { def }}{=}\left\langle\phi_{2 \gamma}(\cdot-\mathrm{Q} \boldsymbol{k}), \phi_{2 \gamma}(\cdot)\right\rangle$ appears in the resulting equation. Using its symmetry, we can restate (26) as follows:

$$
(\breve{h} * a * g)[\mathrm{D} \boldsymbol{k}] \equiv 0
$$

with $\breve{h}[\boldsymbol{k}] \stackrel{\text { def }}{=} h[-\boldsymbol{k}]$.

Let us define $b \stackrel{\text { def }}{=} \breve{h} * a * g$. The above relation then finds the following Fourier domain expression (cf. Viscito and Allebach [55])

$$
\hat{\mathrm{B}}_{\mathrm{Q}}(\boldsymbol{\omega})+\sum_{1 \leq i<|\mathrm{D}|} \hat{\mathrm{B}}_{\mathrm{Q}}\left(\boldsymbol{\omega}-2 \pi \mathrm{Q}^{-\mathrm{T}} \mathrm{D}^{-\mathrm{T}} \boldsymbol{\zeta}_{\boldsymbol{i}}\right)=0 .
$$

From the definition of $b$, we have

$$
\hat{\mathrm{B}}_{\mathrm{Q}}(\boldsymbol{\omega})=\overline{\hat{\mathrm{H}}_{\mathrm{Q}}(\boldsymbol{\omega})} \hat{\mathrm{A}}_{\mathrm{Q}}(\boldsymbol{\omega}) \hat{\mathrm{G}}_{\mathrm{Q}}(\boldsymbol{\omega}) .
$$

Therefore

$$
\hat{\mathrm{G}}_{\mathrm{Q}}(\boldsymbol{\omega})=-\frac{\sum_{1 \leq i<|\mathrm{D}|} \hat{\mathrm{B}}_{\mathrm{Q}}\left(\boldsymbol{\omega}-2 \pi \mathrm{Q}^{-\mathrm{T}} \mathrm{D}^{-\top} \boldsymbol{\zeta}_{\boldsymbol{i}}\right)}{\hat{\mathrm{H}}_{\mathrm{Q}}(\boldsymbol{\omega}) \hat{\mathrm{A}}_{\mathrm{Q}}(\boldsymbol{\omega})} .
$$

We see from (25) and (27) that the numerator has an uncancelled (and isotropic) zero of degree $2 \gamma$ at the origin. Since $\hat{G}(\boldsymbol{\omega})$ is by definition bounded (as $g \in \ell_{1}$ ), this means that we can extract the symbol $\|\boldsymbol{\omega}\|^{2 \gamma}$ (corresponding to $\Delta^{\gamma}$ ) from the Fourier transform of the wavelet filter, and consequently from the Fourier transform of the wavelet itself. In other words, the function

$$
\hat{\eta}(\boldsymbol{\omega}) \stackrel{\text { def }}{=}\|\boldsymbol{\omega}\|^{-2 \gamma} \hat{\psi}_{2 \gamma}(\boldsymbol{\omega})
$$

will be continuous at $\mathbf{0}$.

We also note that the wavelet, by construction, has the same Sobolev regularity as the B-splines; i.e., its Fourier transform decays like $\|\boldsymbol{\omega}\|^{-2 \gamma}$, leading to a $\|\boldsymbol{\omega}\|^{-4 \gamma}$-like decay for $\hat{\eta}(\boldsymbol{\omega})$. From this we deduce that $\eta(\boldsymbol{x})$ is of the claimed Sobolev regularity. That it is also a polyharmonic spline of order $4 \gamma$ follows from the second part of Proposition 2.

\section{APPENDIX II}

\section{PROOF OF THEOREM 4}

As was mentioned in the introduction, the characteristic functional of a Gaussian field $\mathfrak{X}$ satisfies (see Gel'fand et al. [39, ch. III, §2.6])

$$
\mathbf{Z}_{\mathfrak{X}}(u)=\exp \left(-\frac{1}{2}\langle\langle u, u\rangle\rangle_{\mathfrak{X}}\right)
$$

Therefore, in our case, we need to show that for $0<H<1$

$$
\langle\langle u, u\rangle\rangle_{\mathfrak{B}_{H}}=\frac{\epsilon_{H}^{2}}{(2 \pi)^{d}} \int \mathrm{d} \boldsymbol{\omega} \frac{|\hat{u}(\boldsymbol{\omega})-\hat{u}(\mathbf{0})|^{2}}{\|\boldsymbol{\omega}\|^{2 H+d}} .
$$

This correlation form is related to the (generalized) correlation function $c_{\mathfrak{B}_{H}}\left(\boldsymbol{x}, \boldsymbol{x}^{\prime}\right)$ thus [ibid., ch. III, §2.1]

$$
\langle\langle u, v\rangle\rangle_{\mathfrak{B}_{H}}=\int \mathrm{d} \boldsymbol{x} \mathrm{d} \boldsymbol{x}^{\prime} c_{\mathfrak{B}_{H}}\left(\boldsymbol{x}, \boldsymbol{x}^{\prime}\right) u(\boldsymbol{x}) v\left(\boldsymbol{x}^{\prime}\right) .
$$

The correlation function of a normalized fractional Brownian field with parameter $H, 0<H<1$, derived from its variogram, is

$$
c_{\mathfrak{B}_{H}}\left(\boldsymbol{x}, \boldsymbol{x}^{\prime}\right)=\left(\|\boldsymbol{x}\|^{2 H}+\left\|\boldsymbol{x}^{\prime}\right\|^{2 H}-\left\|\boldsymbol{x}-\boldsymbol{x}^{\prime}\right\|^{2 H}\right) .
$$

To show (28), we plug (30) into (29), and break the integral at the additions to get (after replacing $\boldsymbol{x}$ in the first, $\boldsymbol{x}^{\prime}$ in the second, and $\boldsymbol{x}^{\prime}-\boldsymbol{x}$ in the last integral, all by $\boldsymbol{x}$ )

$$
\begin{aligned}
\langle\langle u, u\rangle\rangle_{\mathfrak{B}_{H}}= & \left\langle\|\boldsymbol{x}\|^{2 H}, \mathcal{F}^{-1}\{\overline{\hat{u}(\mathbf{0})} \hat{u}(\boldsymbol{\omega})\}\right\rangle \\
& +\left\langle\|\boldsymbol{x}\|^{2 H}, \mathcal{F}^{-1}\{\overline{\hat{u}(\boldsymbol{\omega})} \hat{u}(\mathbf{0})\}\right\rangle \\
& -\left\langle\|\boldsymbol{x}\|^{2 H}, \mathcal{F}^{-1}\{\overline{\hat{u}(\boldsymbol{\omega})} \hat{u}(\boldsymbol{\omega})\}\right\rangle \\
= & -\left\langle\|\boldsymbol{x}\|^{2 H}, \mathcal{F}^{-1}\{\hat{v}(\boldsymbol{\omega})\}\right\rangle
\end{aligned}
$$

where

$$
\begin{aligned}
\hat{v}(\boldsymbol{\omega}) & \stackrel{\text { def }}{=} \bar{u}(\mathbf{0}) \hat{u}(\boldsymbol{\omega})+\overline{\hat{u}(\boldsymbol{\omega})} \hat{u}(\mathbf{0})-\overline{\hat{u}(\boldsymbol{\omega})} \hat{u}(\boldsymbol{\omega}) \\
& =|\hat{u}(\boldsymbol{\omega})-\hat{u}(\mathbf{0})|^{2}-|\hat{u}(\mathbf{0})|^{2}
\end{aligned}
$$

is a linear combination of test functions and is, therefore, a valid test function itself.

The inner product in (31) can be evaluated in the Fourier domain by applying the Parseval equivalence

$$
\left\langle\|\boldsymbol{x}\|^{2 H}, \mathcal{F}^{-1}\{\hat{v}(\boldsymbol{\omega})\}\right\rangle=-(2 \pi)^{-d}\left\langle\epsilon_{H}^{2} \mathrm{R}\|\boldsymbol{\omega}\|^{-2 H-d}, \hat{v}(\boldsymbol{\omega})\right\rangle
$$

valid for $2 H \neq-d,-d-2, \ldots$ [38, p. 363]. Here $\mathrm{R}\|\boldsymbol{\omega}\|^{-2 H-d}$ is a generalized function (distribution) that corresponds to a particular (canonical) regularization of the function $\|\boldsymbol{\omega}\|^{-2 H-d}$. The canonical regularization is to be conducted according to the recipe given in Gel'fand and Shilov [38, §3.3], as detailed below.

We restate (32) in (hyper)spherical coordinates as

$$
\langle\langle u, u\rangle\rangle_{\mathfrak{B}_{H}}=(2 \pi)^{-d} \epsilon_{H}^{2} \Omega_{d}\left\langle\mathrm{R} \rho^{-2 H-1}, S_{\hat{v}}(\rho)\right\rangle
$$

where $\rho \stackrel{\text { def }}{=}\|\boldsymbol{\omega}\|, \Omega_{d} \stackrel{\text { def }}{=}$ the area of the hypersphere in $\mathbb{R}^{d}$, and $S_{\hat{v}}(\rho)$ denotes the average of $\hat{v}(\boldsymbol{\omega})$ over the hypersphere of radius $\rho$ centered at the origin. Also, $\mathrm{R} \rho^{-2 H-1}$ denotes the particular regularization of $\rho^{-2 H-1}$ invoked in (33).

$S_{\hat{v}}(\rho)$ is a smooth and even function of $\rho$ with rapid decay, with a Taylor series expansion of the form

$$
S_{\hat{v}}(\rho)=\hat{v}(\mathbf{0})+a_{2} \rho^{2}+a_{4} \rho^{4}+\cdots+a_{2 k} \rho^{2 k}+\mathrm{o}\left(\rho^{2 k}\right) .
$$


For $0<H<1$, we have $-3<-2 H-1<0$ and from there, by the definition of the generalized function $\mathrm{R} \rho^{-2 H-1}$ (see [38, p. 363])

$$
\left\langle\mathrm{R} \rho^{-2 H-1}, S_{\hat{v}}(\rho)\right\rangle=\int_{0}^{\infty} \mathrm{d} \rho \rho^{-2 H-1}\left[S_{\hat{v}}(\rho)-\hat{v}(\mathbf{0})\right]
$$

(where the right-hand integral should be interpreted as a limit). By expanding $S_{\hat{v}}(\rho)$ and returning to Cartesian coordinates, we can now write

$$
\begin{aligned}
\left\langle\|\boldsymbol{\omega}\|^{-2 H-d}, \hat{v}(\boldsymbol{\omega})\right\rangle & =\int \mathrm{d} \boldsymbol{\omega}\|\boldsymbol{\omega}\|^{-2 H-d}[\hat{v}(\boldsymbol{\omega})-\hat{v}(\mathbf{0})] \\
& =\int \mathrm{d} \boldsymbol{\omega}\|\boldsymbol{\omega}\|^{-2 H-d}|\hat{u}(\boldsymbol{\omega})-\hat{u}(\mathbf{0})|^{2}
\end{aligned}
$$

(using the definition of $\hat{v}(\boldsymbol{\omega})$ ). From combining this with (32), we arrive at the desired result, i.e., (28).

Remark 6: The following lemma allows us to generalize the results given here for $0<H<1$ to the case of noninteger $H>1$. The proof is technical and is not reproduced here.

Lemma 1: Let $v(\boldsymbol{x})$ be a test function and $H>0$ be noninteger. Then, in the sense of generalized functions of Gel'fand and Shilov

$$
\begin{aligned}
&\left\langle\|\boldsymbol{x}\|^{2 H}, v(\boldsymbol{x})\right\rangle=-\epsilon_{H}^{2} \int \mathrm{d} \boldsymbol{\omega}\|\boldsymbol{\omega}\|^{-2 H-d} \\
& \times\left(\hat{v}(\boldsymbol{\omega})-\Gamma\left(\frac{d}{2}\right) \sum_{0 \leq 2 k \leq\lfloor 2 H\rfloor} \frac{\Delta^{k} \hat{v}(\mathbf{0})\|\boldsymbol{\omega}\|^{2 k}}{2^{2 k} k ! \Gamma\left(k+\frac{d}{2}\right)}\right) .
\end{aligned}
$$

\section{ACKNOWLEDGMENT}

The authors would like to thank K. Balać, who conducted the wavelet analysis of fMRI images. The authors would also like to thank Dr T. Blu and R. Delgado for interesting discussions, as well as the anonymous referees for their detailed and helpful reviews.

\section{REFERENCES}

[1] B. B. Mandelbrot, Gaussian Self-Affinity and Fractals: Globality, The Earth $1 / f$ Noise, and $R / S$. New York: Springer, 2002, vol. H, Selecta (Old or New).

[2] B. B. Mandelbrot, The Fractal Geometry of Nature. New York: W. H. Freeman, 1982.

[3] B. B. Mandelbrot and J. W. Van Ness, "Fractional Brownian motions, fractional noises and applications," SIAM Rev., vol. 10, no. 4, 1968.

[4] P. Chainais, R. Riedi, and P. Abry, "Warped infinitely divisible cascades: Beyond power laws," Traitement du Signal, vol. 22, no. 1, pp. 27-39, 2005.

[5] P. Lévy, Processus stochastiques et mouvement brownien, 2nd ed. France: Gauthier-Villars, 1965.

[6] M. S. Keshner, "1/f noise," Proc. IEEE, vol. 70, no. 3, pp. 212-218, Mar. 1982.

[7] W. E. Leland, W. Willinger, M. S. Taqqu, and D. V. Wilson, "On the self-similar nature of ethernet traffic," SIGCOMM Comput. Commun. Rev., vol. 25, no. 1, pp. 202-213, 1995.

[8] B. Pesquet-Popescu and J. Levy Véhel, "Stochastic fractal models for image processing," IEEE Signal Process. Mag., vol. 19, no. 5, pp. 48-62, May 2002.

[9] A. P. Pentland, "Fractal-based description of natural scenes," IEEE Trans. Pattern Anal. Mach. Intell., vol. PAMI-6, no. 6, pp. 661-674, Nov. 1984.

[10] D. J. Field, "Scale-invariance and self-similar 'wavelet' transforms: An analysis of natural scenes and mamalian visual systems," in Wavelets, Fractals, and Fourier Transforms, M. Farge, J. C. R. Hunt, and J. C. Vassilicos, Eds. Oxford, U.K.: Clarendon, 1993.
[11] N. Sarshar and X. Wu, "On rate-distortion models for natural images and wavelet coding performance," IEEE Trans. Image Process., vol. 16, no. 5, pp. 1383-1394, May 2007.

[12] H. E. Hurst, "Long-term storage capacity of reservoirs," Trans. Amer Soc. Civil Eng., vol. 116, pp. 770-808, 1951.

[13] T. Lundahl, W. J. Ohley, S. M. Kay, and R. Siffert, "Fractional Brownian motion: A maximum likelihood estimator and its application to image texture," IEEE Trans. Med. Imag., vol. MI-5, no. 3, pp. 152-161, Sep. 1986.

[14] B. J. Super and A. C. Bovik, "Localized measurement of image fractal dimension using Gabor filters," J. Vis. Commun. Image Represent., vol. 2, no. 2, pp. 114-128, 1991

[15] S. Mallat, A Wavelet Tour of Signal Processing, 2nd ed. San Diego, CA: Academic, 1999.

[16] M. Unser and T. Blu, "Wavelet theory demystified," IEEE Trans. Signal Process., vol. 51, no. 2, pp. 470-483, Feb. 2003.

[17] A. Arneodo, G. Grasseau, and M. Holschneider, "Wavelet transform of multifractals," Phys. Rev. Lett., vol. 61, no. 20, Nov. 1988.

[18] S. Mallat, "A theory for multiresolution signal decomposition: The wavelet representation," IEEE Trans. Pattern Anal. Mach. Intell., vol. 11, no. 7, pp. 673-693, Jul. 1989.

[19] P. Flandrin, "On the spectrum of fractional Brownian motions," IEEE Trans. Inf. Theory, vol. 35, no. 1, pp. 197-199, Jan. 1989.

[20] P. Flandrin, "Wavelet analysis and synthesis of fractional Brownian motion," IEEE Trans. Inf. Theory, vol. 38, no. 2, pp. 910-916, Mar. 1992.

[21] M. Farge, "Wavelet transforms and their applications to turbulence," Annu. Rev. Fluid Mech., vol. 24, pp. 395-457, 1992.

[22] G. W. Wornell and A. V. Oppenheim, "Estimation of fractal signals from noisy measurements using wavelets," IEEE Trans. Signal Process., vol. 40, no. 3, pp. 611-623, Mar. 1992.

[23] Y. Meyer, F. Sellan, and M. Taqqu, "Wavelets, generalized white noise and fractional integration: The synthesis of fractional Brownian motion," J. Fourier Anal. Appl., vol. 5, no. 5, pp. 465-494, 1999.

[24] Y. Meyer, Wavelets, Vibrations and Scalings. Providence, RI: Amer Math. Soc., 1997, vol. 9, CRM Monograph Series.

[25] A. H. Tewfik and M. Kim, "Correlation structure of the discrete wavelet coefficients of fractional Brownian motion," IEEE Trans. Inf. Theory, vol. 38, no. 2, pp. 904-909, Mar. 1992.

[26] E. Masry, "The wavelet transform of stochastic processes with stationary increments and its application to fractional Brownian motion," IEEE Trans. Inf. Theory, vol. 39, no. 1, pp. 260-264, Jan. 1993.

[27] G. W. Wornell, "Wavelet-based representations for the $1 / f$ family of fractal processes," Proc. IEEE, vol. 81, no. 10, pp. 1428-1450, Oct. 1993.

[28] D. Veitch and P. Abry, "A wavelet-based joint estimator of the parameters of long-range dependence," IEEE Trans. Inf. Theory, vol. 45, no. 3, pp. 878-897, Apr. 1999.

[29] B. Ninness, "Estimation of $1 / f$ noise," IEEE Trans. Inf. Theory, vol. 44, no. 1, pp. 32-46, Jan. 1998.

[30] T. Blu and M. Unser, "Self-similarity: Part II-Optimal estimation of fractal processes," IEEE Trans. Signal Process., vol. 55, no. 4, pp. 1364-1378, Apr. 2007.

[31] A. Benassi, S. Jaffard, and D. Roux, "Elliptic gaussian random processes," Rev. Mat. Iberoamer., vol. 13, no. 1, pp. 19-90, 1997.

[32] A. Benassi and J. Istas, "Processus autosimilaires," in Lois d'échelle, fractales et ondelettes, P. Abry, P. Gonçalvès, and J. Levy Véhel, Eds. Paris, France: Hermes Lavoisier, 2002, vol. 2, Traité IC2.

[33] W. Wyss, "Fractional noise," Found. Phys. Lett., vol. 4, pp. 235-246, 1991.

[34] M. Unser and T. Blu, "Self-similarity: Part I—Splines and operators," IEEE Trans. Signal Process., vol. 55, no. 4, pp. 1352-1363, Apr. 2007.

[35] C. Rabut, "Elementary m-harmonic cardinal B-splines," Numer. Alg. vol. 2, pp. 39-61, 1992.

[36] B. Bacchelli, M. Bozzini, C. Rabut, and M.-L. Varas, "Decomposition and reconstruction of multidimensional signals using polyharmonic pre-wavelets," Appl. Comput. Harmon. Anal., vol. 18, no. 3, pp. 282-299, May 2005.

[37] D. Van De Ville, T. Blu, and M. Unser, "Isotropic polyharmonic B-splines: Scaling functions and wavelets," IEEE Trans. Image Process., vol. 14, no. 11, pp. 1798-1813, Nov. 2005.

[38] I. M. Gel'fand and G. E. Shilov, Properties and Operations Transl. :Translated by Eugene Saletan. New York: Academic, 1964, vol. I, Generalized Functions.

[39] I. M. Gel'fand and N. Vilenkin, Applications of Harmonic Analysis Transl.:Translated by Amiel Feinstein. New York: Academic, 1964, vol. IV, Generalized Functions. 
[40] J. H. Conway and N. J. A. Sloane, Sphere Packings, Lattices and Groups. New York: Springer-Verlag, 1988.

[41] J. D. Gibson and K. Sayood, "Lattice quantization," Adv.Electron. Electron Phys., vol. 72, pp. 259-330, 1988.

[42] J. Kybic, T. Blu, and M. Unser, "Generalized sampling: A variational approach-Part I: Theory," IEEE Trans. Signal Process., vol. 50, no. 8, pp. 1965-1976, Aug. 2002.

[43] J. Duchon, "Splines minimizing rotation-invariant semi-norms in Sobolev spaces," in Proc. Constructive Theory of Functions of Several Variables, A. Dold and B. Eckmann, Eds. New York: Springer-Verlag, 1977, Lecture Notes in Mathematics, pp. 85-100.

[44] M. Arigovindan, "Variational Reconstruction of Vector and Scalar Images from Non-Uniform Samples," Ph.D. dissertation, EPFL, Biomedical Imaging Group, Lausanne, Switzerland, 2005.

[45] I. M. Gel'fand and G. E. Shilov, Spaces of Fundamental and Generalized Functions Transl.:Translated by Morris D. Friedman. New York: Academic, 1968, vol. II, Generalized Functions.

[46] M. Unser and T. Blu, "Fractional splines and wavelets," SIAM Rev., vol. 42, no. 1, pp. 43-67, Mar. 2000.

[47] C. Micchelli, C. Rabut, and F. I. Utreras, "Using the refinement equation for the construction of pre-wavelets III: Elliptic splines," Numer. Alg., vol. 1, no. 3, pp. 331-352, 1991.

[48] R. L. Dobrushin, "Gaussian and their subordinated self-similar random generalized fields," Ann. Probab., vol. 7, no. 1, pp. 1-28, 1979.

[49] J.-P. Kahane, Some Random Series of Functions, 2nd ed. Cambridge, U.K.: Cambridge Univ. Press, 1985.

[50] T. Lindstrøm, "Fractional Brownian fields as integrals of white noise," Bull. London Math. Soc., vol. 25, no. 1, pp. 83-88, 1993.

[51] G. Samorodnitsky and M. S. Taqqu, Stable Non-Gaussian Random Processes: Stochastic Models with Infinite Variance. New York: Chapman \& Hall, 1994.

[52] E. Bullmore, M. Brammer, I. Harvey, R. Persaud, R. Murray, and M. Ron, "Fractal analysis of the boundary between white matter and cerebral cortex in magnetic resonance images: A controlled study of schizophrenic and manic-depressive patients," Psychol. Med., vol. 24, no. 3, pp. 771-781, 1994.

[53] S. Heinzer, T. Krucker, M. Stampanoni, R. Abela, E. P. Meyer, A. Schuler, P. Schneider, and R. Muller, "Hierarchical microimaging for multiscale analysis of large vascular networks," NeuroImage, vol. 32, no. 2 , pp. 626-636, 2006 .

[54] M. N. Kolountzakis, "The study of translational tiling with Fourier analysis," in Fourier Analysis and Convexity, L. Brandolini, L. Colzani, A. Iosevich, and G. Travaglini, Eds. Boston, MA: Birkhäuser, 2004, Applied and Numerical Harmonic Analysis, pp. 131-187.

[55] E. Viscito and J. P. Allebach, "The analysis and design of multidimensional FIR perfect reconstruction filter banks for arbitrary sampling lattices," IEEE Trans. Circuits Syst., vol. 38, no. 1, pp. 29-41, Jan. 1991.

[56] W. R. Madych and S. A. Nelson, "Polyharmonic cardinal splines," $J$. Approx. Theory, vol. 50, no. 2, pp. 141-156, Feb. 1990.

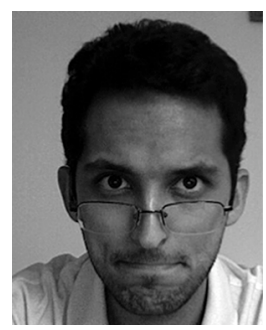

Pouya Dehghani Tafti (M'00) was born in 1981. He received the B.Sc. degree from Sharif University of Technology, Tehran, Iran, and the M.A.Sc. degree from McMaster University, Hamilton, ON, Canada, both in electrical engineering. He is currently pursuing the Ph.D. degree in the Biomedical Imaging Group, École Polytechnique Fédérale de Lausanne (EPFL), Switzerland.

His research interests include stochastic modeling and mathematical signal processing.

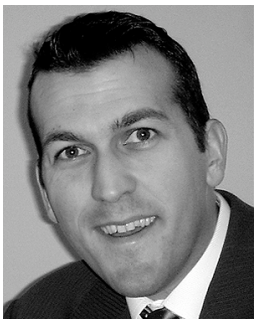

Dimitri Van De Ville (M'02) received the M.S. degree in engineering and computer sciences from Ghent University, Ghent, Belgium, in 1998, and the Ph.D. degree in 2002.

He received a grant as Research Assistant with the Fund for Scientific Research Flanders Belgium (FWO). In 2002, he joined Prof. M. Unser's Biomedical Imaging Group at the École Polytechnique Fédérale de Lausanne (EPFL), Lausanne, Switzerland. Since December 2005, he has been heading the Signal Processing Unit at the University Hospital of Geneva, Geneva, Switzerland, as part of the Centre d'Imagerie Biomédicale (CIBM). His research interests include wavelets, statistical analysis, multidimensional splines, and applications in biomedical imaging, such as functional magnetic resonance imaging, spectroscopy, electro-encephalography, and microscopy.

Dr. Van De Ville serves as an Associate Editor for the IEEE TRANSACTIONS ON IMAGE PROCESSING (since February 2006) and was previously an Associate Editor for IEEE SIGNAL PROCESSING LETTERS (2004-2006). Since 2003, he has also been an Editor and Webmaster of The Wavelet Digest. He is co-chair of the Wavelets XII (2007) Wavelets XIII (2009) international conferences, together with V. Goyal and M. Papadakis.

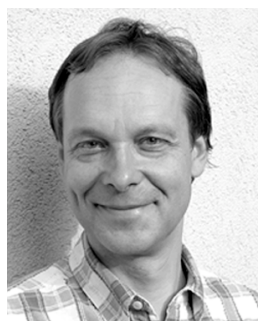

Michael Unser (M'89-SM'94-F'99) received the M.S. (summa cum laude) and Ph.D. degrees in electrical engineering in 1981 and 1984, respectively, from the École Polytechnique Fédérale de Lausanne (EPFL), Switzerland.

From 1985 to 1997, he was a Scientist with the National Institutes of Health, Bethesda, MD. He is now full Professor and Director of the Biomedical Imaging Group, EPFL. His main research area is biomedical image processing. He has a strong interest in sampling theories, multiresolution algorithms, wavelets, and the use of splines for image processing. He has published over 150 journal papers on those topics and is one of ISI's Highly Cited authors in Engineering (http://isihighlycited.com).

Dr. Unser has held the position of associate Editor-in-Chief (2003-2005) for the IEEE TRANSACTIONS ON MEDICAL IMAGING and has served as Associate Editor for the same journal (1999-2002; 2006-2007), the IEEE Transactions on IMAge Processing (1992-1995), and the IEEE Signal PROCESSING LETTERS (1994-1998). He is currently member of the editorial boards of Foundations and Trends in Signal Processing, the SIAM Journal of Imaging Sciences, and Sampling Theory in Signal and Image Processing. He co-organized the first IEEE International Symposium on Biomedical Imaging (ISBI2002). He was the founding chair of the technical committee of the IEEE-SP Society on Bio Imaging and Signal Processing (BISP). He received the 1995 and 2003 Best Paper Awards and the 2000 Magazine Award from the IEEE Signal Processing Society. 